\title{
20. EVOLUTION OF PLIOCENE CLIMATE CYCLICITY AT HOLE 806B (5-2 Ma): OXYGEN ISOTOPE RECORD ${ }^{1}$
}

\author{
Eystein Jansen, ${ }^{2}$ Larry A. Mayer, ${ }^{3}$ Jan Backman, ${ }^{4}$ R. Mark Leckie,${ }^{5}$ and Toshiaki Takayama ${ }^{6}$
}

\begin{abstract}
A detailed Pliocene oxygen isotope record from the Ontong Java Plateau, based on measurements of the surface-dwelling planktonic foraminifer Globigerinoides sacculifer, was produced for the period from 5 to $2 \mathrm{Ma}$. The record documents major long- and short-term climate changes. The results show periods of enhanced ice volume at 4.6 to $4.3 \mathrm{Ma}$ and after $2.85 \mathrm{Ma}$, a long-term warming trend from 4.1 to $3.7 \mathrm{Ma}$, and a distinct cooling trend that was initiated at $3.5 \mathrm{Ma}$ and progressed through the initiation of large-scale Northern Hemisphere glaciation after $2.85 \mathrm{Ma}$ (according to the time scale of Shackleton and others proposed in 1990). Periods of high average ice volumes also show the highest $\delta^{18} \mathrm{O}$ amplitudes. The pattern of climate cyclicity changed markedly at about $2.85 \mathrm{Ma}$. Earlier times were marked by high-frequency variability at the precessional frequencies or even higher frequencies, pointing to low-latitude processes as a main controlling factor driving planktonic $\delta^{18} \mathrm{O}$ variability in this period. The high-frequency variability is not coherent with insolation and points to strong nonlinearity in the way the climate system responded to orbital forcing before the onset of large scale Northern Hemisphere glaciation. After $3 \mathrm{Ma}$, stronger 41-k.y. cyclicity appears in the record. The shift in pattern is clearest around $2.85 \mathrm{Ma}$ (according to the time scale proposed by Shackleton and others in 1990), 100-200 k.y. before the most dramatic spread of Northern Hemisphere ice sheets. This indicates that high-latitude processes from this point on began to take over and influence most strongly the $\delta^{18} \mathrm{O}$ record, which now reflects ice-volume fluctuations related to the climatic effects of obliquity forcing on the seasonality of high-latitude areas, most probably in the Northern Hemisphere. The general Pliocene trend is that high-latitude climate sensitivity and instability was increasing, and the causal factors producing the intensified glacial cyclicity during the Pliocene must be factors that enhance cooling and climate sensitivity in the subarctic areas.
\end{abstract}

\section{INTRODUCTION}

The Ontong Java Plateau is a classic area for stable isotope work; many of the pioneering studies of Quaternary oxygen isotope stratigraphy are based on material from this area (Shackleton and Opdyke, 1973; Berger et al., 1978; Imbrie et al., 1984). This region has been the focus of these studies because continuous carbonate accumulation above the lysocline, in an equatorial setting, has provided high-quality material for isotope analyses. In addition, the deep thermocline of the western equatorial Pacific and low seasonality makes this an area of limited local temperature overprint and thus a location where a global climate record can be extracted from the oxygen isotopic composition of surface-dwelling planktonic foraminifers.

The extraction of a high-resolution global climate record from the Ontong Java Plateau should enable us to study the evolution of the climate system in detail. Here we focus on the interval from 5 to $2 \mathrm{Ma}$, which represents an important transitional period in the evolution of the global climate. It spans the interval that starts after the upper Miocene Messinian stage, which was characterized by regressions and increases in ice volume, and which includes the Pliocene phases of relatively low ice volumes and the cooling that resulted in the emergence of major glacial-interglacial climate cycles in the late Pliocene and the Pleistocene. With a detailed record, we aim to shed light upon the long-term trends in the climate record and the variability of the record; in particular, we will address how the sensitivity of

\footnotetext{
${ }^{1}$ Berger, W.H., Kroenke, L.W., Mayer, L.A., et al., 1993. Proc. ODP, Sci. Results, 130: College Station, TX (Ocean Drilling Program).

${ }^{2}$ Department of Geology, Section B, Bergen University, Allégaten 41, N-5007 Bergen, Norway.

${ }^{3}$ Ocean Mapping Group, Department of Surveying Engineering, University of New Brunswick, P.O. Box 4400, Fredericton, New Brunswick, Canada E3B 5A3.

${ }^{4}$ Department of Geology, Stockholm University, S-10691 Stockholm, Sweden.

${ }^{5}$ Department of Geology and Geography, University of Massachusetts, Morrill Science Center, Amherst, MA 01003, U.S.A.

${ }^{6}$ Department of Geology, College of Liberal Arts, Kanazawa University, 1-1 Marunochi, Kanazawa 920, Japan.
}

the climate system responded to orbital or Milankovitch forcing during this transition. It was previously documented through detailed oxygen isotope stratigraphy that the climate system's response (in terms of ice volume) to the Milankovitch forcing function has varied in terms of amplitude and frequency and has shifted from being a relatively pure $41-\mathrm{k}$.y. response at $2-1 \mathrm{Ma}$ to a complex pattern of dominant 100-k.y. glacial cyclicity, with additional variance concentrated at the 23-k.y. precessional frequency and the 41-k.y. obliquity frequency during the last $1 \mathrm{~m} . \mathrm{y}$. of Earth's history (Ruddiman et al., 1986, 1989; Shackleton et al., 1990). Our aim is to bring the analysis of the frequency components of global ice volumes further back in time and evaluate the patterns of climate fluctuations before and during the onset of major Northern Hemisphere glaciations, and to investigate to what extent the evolution of stronger glacial variations during the Pliocene was accompanied by specific changes in the pattern of climate variablity.

For this study, we chose Hole $806 \mathrm{~B}$, cored at $0^{\circ} 19.11^{\prime} \mathrm{N}$, $159^{\circ} 21.69^{\prime} \mathrm{E}$ in a water depth of $2520 \mathrm{~m}$. The cores were sampled at $10-\mathrm{cm}$ intervals except where prohibited by routine whole-round sampling. The location of Site 806 on the shallower part of the plateau, almost on the equator and above the regional calcite lysocline, ensures high sedimentation rates and minimal dissolution effects. We studied the section between 45 and $155 \mathrm{~m}$. Based on the biostratigraphic age information, this should cover major portions of the Pliocene, starting in the lower Pliocene after the end of the Messinian, and includes the inception of large-scale glaciation in the Northern Hemisphere at about 2.5 Ma (Shackleton et al., 1984; Jansen et al., 1988; Jansen and Sjøholm, 1991).

\section{METHODS}

The isotope record was produced from Globigerinoides sacculifer: Measurements were done on specimens in the $355-425 \mu \mathrm{m}$ fraction. About ten individuals were picked for each measurement; all were specimens with a saclike final chamber. We avoided measuring the related species Globigerinoides fistulosus. The samples 
were ultrasonically cleaned in methanol, roasted in vacuo at $380^{\circ} \mathrm{C}$ for $40 \mathrm{~min}$. Gas for isotope measurements was produced by reaction with orthophosphoric acid at $50^{\circ} \mathrm{C}$ in an automated on-line system with acid added to the samples in individual reaction chambers. The isotope measurements were performed on a Finnegan MAT 251 mass spectrometer at Bergen University. Results are reported with respect to the PDB standard through calibration against NBS 19 and 20 standards. The reproducibility of the system is $\pm 0.06 \%$, based on replicate measurements of an internal carbonate standard.

Globigerinoides sacculifer has been shown to be a surface-dwelling foraminifer (Fairbanks et al., 1982; Ravelo, 1991), which implies that isotope values will reflect the combined effect of the isotopic composition of the western equatorial Pacific surface water and the surface-water temperature. As noted earlier by Shackleton and Opdyke (1973), the deep thermocline and the stable warm surfacewater temperature in this region should minimize the local temperature effect on the isotope record and emphasize the variability that stems from variations in global ocean water $\delta^{18} \mathrm{O}$ (i.e., from variations in global ice volume).

As a first step toward producing a composite depth section for Site 806, we adjusted the depths of Hole 806B to the spliced depth scale we developed using GRAPE data in the accompanying paper by Mayer et al. (in press). Thus, all data are plotted against this spliced depth scale and reported as meters composite depth (MCD). Raw data are given in Table 1 against the original depth in Hole $806 \mathrm{~B}$ as well as the spliced depth (MCD). Based on the nannofossil biostratigraphy age picks (Fig. 1), this was converted to age using a preliminary age scale. Second, as discussed by Mayer et al. (this volume), we used the recognition of isotope stages in the period from 2.4 to $3 \mathrm{Ma}$ to improve the age model. The data were converted to the age domain using the same age model based on datums from Shackleton et al. (1990) and N.J. Shackleton (pers. comm., 1992) that was employed by Mayer et al. (this volume). The data are plotted vs. age using this age model. Some ages from the biostratigraphic age model are given in parenthesis in the following text to make comparisons easier. When comparing ages from previous studies, it should be kept in mind that these are normally obtained from standard magneto- and biostratigraphic time scales and are younger than comparable ages from this study by approximately $5 \%$.

The composite section that is produced is as complete as possible at the current stage. Further progress can be made by improving the continuity at core breaks by splicing our record with samples from the other holes at this site, and by refining the resolution of some of the biostratigraphic datums through closer sampling. Tuning the record to orbital tuning targets is planned as a second stage. The final result will still be hampered, however, by the fact that the overlap between core breaks is too low in many parts of the section. For this reason, we cannot be completely sure that the isotope record developed from Hole $806 \mathrm{~B}$ is or can be absolutely complete and that all isotope events are represented throughout the $3 \mathrm{~m} . \mathrm{y}$. covered by our record. We feel confident, nevertheless, that we have a record that, because of the very high sedimentation rates in the early and middle Pliocene and the high sampling density, shows a high degree of detail with enough resolution to resolve frequency components at higher frequencies than the high-frequency end of the Milankovitch frequency bands ( 19 and 23 k.y. cycles). The sampling rate gives an average resolution between 2000 and $3000 \mathrm{yr}$.

Figure 1 shows the $G$. sacculifer record plotted vs. depth. The positions of nannofossil and foraminifer datums with respect to the oxygen isotope record are indicated. This is done to facilitate further calibration and correlation of biostratigraphic datums by tying them to detailed oxygen isotope records. It appears as if many of the datums fall on $\delta^{18} \mathrm{O}$ enrichments (i.e., on episodes of global climate cooling). The datums used to construct the initial biostratigraphic time scale are highlighted. Further documentation of biostratigraphic datums can be found in other chapters in this volume.

\section{RESULTS AND DISCUSSION}

Figure 2 shows the oxygen isotope record plotted vs. age. The solid line shows a polynomial best fit to the data, intended to document the major trends in the data set. No unidirectional trend is present in the data, indicating that the long-term climate evolution is not a case of a clear cooling trend from 5 to $2 \mathrm{Ma}$, and that the processes forcing this evolution did not produce a net unidirectional forcing function. Because the low seasonality and thick mixed layer in the western equatorial Pacific should reduce the influence of local temperature variations, we infer as a first approximation that the record chiefly reflects global average ice volume. (High $\delta^{18} \mathrm{O}$ implies larger ice volume.) Consequently, we think that temperature plays a secondary role. Based on this approach, we note periods of relatively high ice volumes, with $\delta^{18} \mathrm{O}$ in excess of $+1 \%$, at $4.6-4.3 \mathrm{Ma}$, and again and more consistently after $2.85(2.73) \mathrm{Ma}$. It is widely thought that large-scale glaciation in the Northern Hemisphere was initiated at about $2.5 \mathrm{Ma}$ (using a standard magnetic time scale), reflecting a change of climate sensitivity superimposed on a general cooling trend (Shackleton et al., 1984; Jansen et al., 1988; Raymo et al., 1989; Jansen and Sjøholm, 1991). Yet the Hole 806B record indicates that global ice volumes similar in magnitude to the interval after $2.5 \mathrm{Ma}$ were present also in the interval from 4.6 to $4.3 \mathrm{Ma}$. As can be seen in Figures 2 and 3, the general $\delta^{18} \mathrm{O}$ values during this period are within the range of the late Quaternary (Berger et al., this volume), thus indicating that the glacial episodes from 4.6 to 4.3 Ma had higher ice volumes than now. Similar episodes are also noticeable in the record from Site 807 (Prentice et al., this volume).

Jansen et al. (1990) and Jansen and Sjøholm (1991) also suggested the existence of intervals in the Pliocene with ice volumes in excess of the present, based on a less well dated and lower resolution benthic oxygen isotope record from the Norwegian Sea. Because of the lack of major ice-raft deposition in the North Atlantic region before $2.57 \mathrm{Ma}$ (Jansen and Sjøholm, 1991), these early $\delta^{18} \mathrm{O}$ enrichments were probably not caused by intensified Northern Hemisphere glaciation. The most likely interpretation is that the $\delta^{18} \mathrm{O}$ enrichments from 4.6 to 4.3 Ma primarily reflect Antarctic glaciation. If we calculate on the basis that no sea-surface temperature (SST) affects the record, and that no significant ice volume was located in the Northern Hemisphere, the ice contained in this ice volume is about 1.5 to 2 times the present Antarctic ice volume (depending on the $\delta^{18} \mathrm{O}$ value in the ice). This is most likely too large of a volume, and the $\delta^{18} \mathrm{O}$ enrichments may indicate a combination of higher global ice volume and lower local SST. Southern ocean records record this time as a relatively warm phase (Hodell and Venz, in press), indicating that the cause for enhanced Antarctic glaciation was more precipitation derived from a warmer ocean. We also note that only the lightest $\delta^{18} \mathrm{O}$ peaks display values as light as those of the Holocene, reported by Berger et al. (this volume) from Site 806 . This indicates that the general SST was a few degrees lower than at present, or that global ice volumes were in excess of the present.

The periods $4.8-4.6$ and 3.9-3.7 Ma were characterized by relatively less ice volume but not less than the Holocene, apparently (Fig. 3). The phases of reduced Pliocene ice extent in Antarctica recorded by Webb and Harwood (1991) are not easily identifiable in the isotope record from Hole $806 \mathrm{~B}$, which has no clear periods with lower isotope values than in the Holocene. This indicates that a general lowering of SST occurred during the Pliocene in the western equatorial Pacific in comparison with the Holocene.

A general trend toward lower ice volumes occurred between 4.3 and $4 \mathrm{Ma}$, and a distinct long-term cooling trend toward higher ice volumes started at about $3.7 \mathrm{Ma}$. This appears to be the cooling trend that led to the inception of large-scale Northern Hemisphere glaciation, which is reflected in an onset of widespread sedimentation of glacial marine deposits in the North Atlantic (Shackleton et al., 1984; Raymo et al., 1989; Jansen et al., 1988; Jansen and Sjøholm, 1991). 


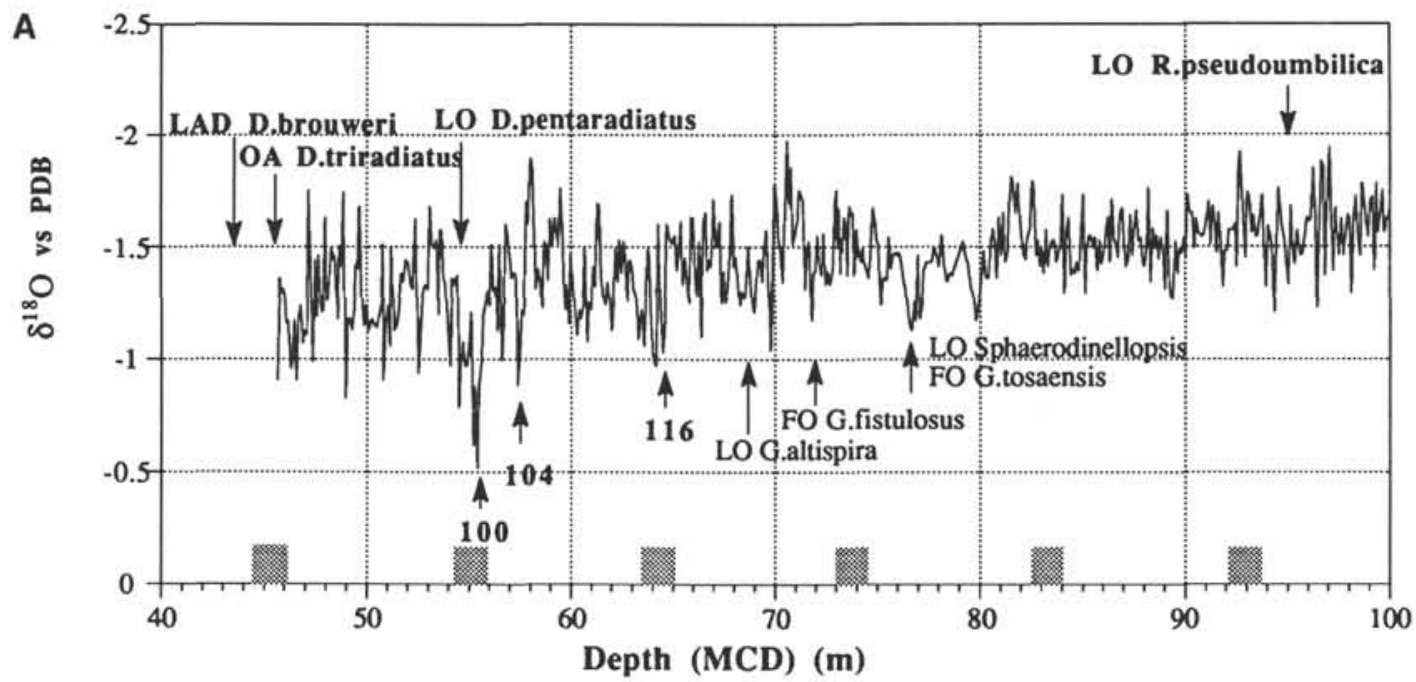

B

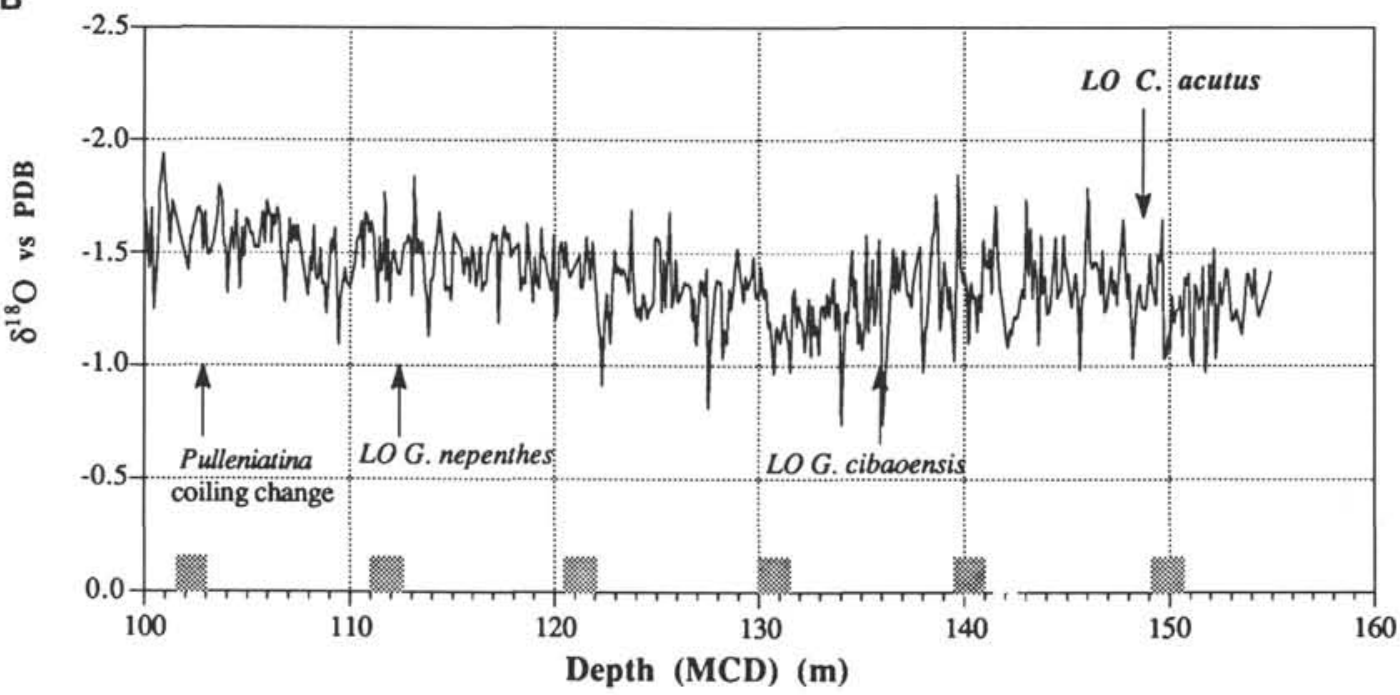

Figure 1. Oxygen isotope record of $G$. sacculifer from Hole 806B plotted vs. spliced depth (MCD) from 40 to $160 \mathrm{~m}$. Biostratigraphic datums are plotted to mark positions on oxygen isotope record. Oxygen isotope stages refer to stage designations by Raymo et al. (1989). The datums in boldface are the ones used for constructing the age model. See accompanying paper by Mayer et al. (this volume). Section 1 of each core is indicated by bars on depth axis.

Superimposed on these long-term trends were higher frequency variability with noticeable changes both in amplitude and periodicity as time evolved. The oldest and youngest parts of the record are similar in some respects. The average $\delta^{18} \mathrm{O}$ was similar, and about $0.6 \%$ higher than in the middle portion of the record. In the intervals before $4.3 \mathrm{Ma}$ and after $2.85 \mathrm{Ma}$, the amplitude of $\delta^{18} \mathrm{O}$ variability was about $0.75 \%$, reaching $1 \%$ or more after $2.6 \mathrm{Ma}$, compared with a general amplitude around $0.5 \%$ between 4 and $3 \mathrm{Ma}$. In comparison, late Quaternary ice-volume changes were about 1.3\%o (Chapell and Shackleton, 1986; Labeyrie et al., 1987; Fairbanks, 1989). Late Quaternary $\delta^{18} \mathrm{O}$ amplitudes recorded in Hole $806 \mathrm{~B}$ are $1.2 \% 0-1.4 \%$ (Berger et al., this volume). It is not straightforward to directly compare ice volumes based on $\delta^{18} \mathrm{O}$ amplitudes because the oxygen isotopic composition of the ice sheets may have varied substantially with time, depending on the air temperature over the ice. Regardless of the absolute magnitude of the ice-volume fluctuations, there appears to be a connection between the average ice volume $\left(\delta^{18} \mathrm{O}\right)$ and the amplitude of $\delta^{18} \mathrm{O}$ variability, indicating that higher amplitudes occur in periods when average ice volumes were large, and a more stable situation with less amplitude was present when global climates ameliorated and there were smaller volumes of continental ice. Figure 2 also indicates that the young part of the record shows tendencies for clearer cyclicity, as compared with the middle part. This will be discussed further in the following sections.

Pronounced isotope enrichments, which reflect episodes of particularly strong glaciation (values exceeding $\div \%$ ) are evident between 4.6 to $4.3 \mathrm{Ma}$. Intermediate-scale $\delta^{18} \mathrm{O}$ increases appear between 3.3 and $3 \mathrm{Ma}$ in accordance with a number of previous studies, pointing to cooling events at this time (Backman and Pestiaux, 1986; Keigwin, 1987; Raymo et al., 1987; Joyce et al., 1990). The next phase of large glacials started at $2.85(2.73) \mathrm{Ma}$, and signals the shift to a different mode of $\delta^{18} \mathrm{O}$ variability. Most noticeable is the glacial at $2.55 \mathrm{Ma}$, which we determine to be isotope Stage 100 based on its amplitude and its position just below the last occurrence (LO) of the Discoaster pentaradiatus datum (Fig. 1) (see discussion of time scales in the accompanying paper by Mayer et al. [this volume]). The isotope values in this stage are similar to those of the largest late Quaternary 
Table 1. Oxygen isotope data measured on the planktonic foraminifer Globigerinoides sacculifer in Hole 806B.

\begin{tabular}{|c|c|c|c|c|c|c|c|c|c|c|c|}
\hline $\begin{array}{l}\text { Depth } \\
\text { (mbsf) }\end{array}$ & $\begin{array}{l}\text { Splice depth (m) } \\
\text { (MCD) }\end{array}$ & $\delta^{18} \mathrm{O}$ & $\begin{array}{l}\text { Depth } \\
\text { (mbsf) }\end{array}$ & $\begin{array}{l}\text { Splice depth (m) } \\
\text { (MCD) }\end{array}$ & $\delta^{18} \mathrm{O}$ & $\begin{array}{l}\text { Depth } \\
\text { (mbsf) }\end{array}$ & $\begin{array}{l}\text { Splice depth (m) } \\
\text { (MCD) }\end{array}$ & $\delta^{18} \mathrm{O}$ & $\begin{array}{l}\text { Depth } \\
\text { (mbsf) }\end{array}$ & $\begin{array}{l}\text { Splice depth }(\mathrm{m}) \\
(\mathrm{MCD})\end{array}$ & $8^{18} \mathrm{O}$ \\
\hline 44.520 & 45.660 & -0.91 & 52.640 & 52.863 & -1.33 & 60.420 & 60.490 & -1.18 & 68.220 & 68.290 & -1.24 \\
\hline 44.620 & 45.760 & -1.36 & 52.750 & 52.991 & -1.31 & 60.520 & 60.590 & -1.24 & 68.320 & 68.390 & -1.30 \\
\hline 44.720 & 45.860 & -1.29 & 52.830 & 53.084 & -1.68 & 60.620 & 60.690 & -1.49 & 68.420 & 68.490 & -1.27 \\
\hline 44.840 & 45.980 & -1.31 & 52.920 & 53.188 & -1.51 & 60.720 & 60.790 & -1.08 & 68.520 & 68.590 & -1.29 \\
\hline 44.920 & 46.060 & -1.16 & 53.010 & 53.292 & -1.49 & 60.820 & 60.890 & -1.25 & 68.620 & 68.690 & -1.50 \\
\hline 45.020 & 46.160 & -1.17 & 53.130 & 53.428 & -1.53 & 60.920 & 60.990 & -1.22 & 68.720 & 68.790 & -1.39 \\
\hline 45.140 & 46.280 & -0.96 & 53.230 & 53.511 & -1.20 & 61.020 & 61.090 & -1.41 & 68.820 & 68.890 & -1.25 \\
\hline 45.250 & 46.390 & -1.01 & 53.320 & 53.585 & -1.57 & 61.120 & 61.190 & -1.32 & 68.920 & 68.990 & -1.21 \\
\hline 45.330 & 46.470 & -1.17 & 53.440 & 53.684 & -1.57 & 61.220 & 61.290 & -1.69 & 69.020 & 69.090 & -1.32 \\
\hline 45.420 & 46.560 & -0.91 & 53.520 & 53.751 & -1.37 & 61.320 & 61.390 & -1.68 & 69.120 & 69.190 & -1.44 \\
\hline 45.510 & 46.650 & -1.18 & 53.620 & 53.833 & -1.33 & 61.420 & 61.490 & -1.37 & 69.220 & 69.290 & -1.44 \\
\hline 45.630 & 46.770 & -1.24 & 53.840 & 54.015 & -1.08 & 61.520 & 61.590 & -1.28 & 69.320 & 69.390 & -1.34 \\
\hline 45.730 & 46.870 & -1.07 & 53.920 & 54.081 & -0.97 & 61.620 & 61.690 & -1.24 & 69.420 & 69.490 & -1.49 \\
\hline 45.820 & 46.960 & -1.13 & 54.020 & 54.164 & -1.36 & 61.720 & 61.790 & -1.34 & 69.520 & 69.590 & -1.57 \\
\hline 45.940 & 47.080 & -1.11 & 54.140 & 54.263 & -1.33 & 61.820 & 61.890 & -1.26 & 69.620 & 69.690 & -1.28 \\
\hline 46.020 & 47.160 & -1.75 & 54.250 & 54.354 & -1.36 & 61.920 & 61.990 & -1.13 & 69.720 & 69.790 & -1.04 \\
\hline 46.120 & 47.260 & -1.37 & 54.320 & 54.412 & -1.35 & 62.020 & 62.090 & -1.24 & 69.820 & 69.890 & -1.38 \\
\hline 46.220 & 47.360 & -0.99 & 54.340 & 54.428 & -1.37 & 62.120 & 62.190 & -1.49 & 69.920 & 69.990 & -1.77 \\
\hline 46.340 & 47.480 & -1.44 & 54.390 & 54.470 & -1.11 & 62.220 & 62.290 & -1.34 & 70.020 & 70.090 & -1.70 \\
\hline 46.420 & 47.560 & -1.19 & 54.420 & 54.494 & -0.79 & 62.320 & 62.390 & -1.53 & 70.120 & 70.190 & -1.54 \\
\hline 46.520 & 47.660 & -1.46 & 54.520 & 54.577 & -0.96 & 62.420 & 62.490 & -1.38 & 70.220 & 70.290 & -1.47 \\
\hline 46.640 & 47.780 & -1.21 & 54.620 & 54.690 & -1.08 & 62.520 & 62.590 & -1.52 & 70.320 & 70.390 & -1.34 \\
\hline 46.750 & 47.890 & -1.22 & 54.720 & 54.790 & -0.97 & 62.620 & 62.690 & -1.43 & 70.420 & 70.490 & -1.63 \\
\hline 46.830 & 47.970 & -1.63 & 54.820 & 54.890 & -0.97 & 62.720 & 62.790 & -1.26 & 70.520 & 70.590 & -1.97 \\
\hline 46.920 & 48.060 & -1.26 & 54.920 & 54.990 & -1.05 & 62.820 & 62.890 & -1.44 & 70.620 & 70.690 & -1.69 \\
\hline 47.010 & 48.150 & -1.28 & 55.020 & 55.090 & -1.21 & 62.920 & 62.990 & -1.41 & 70.720 & 70.790 & -1.85 \\
\hline 47.130 & 48.270 & -1.50 & 55.120 & 55.190 & -0.62 & 63.020 & 63.090 & -1.31 & 70.820 & 70.890 & -1.51 \\
\hline 47.230 & 48.370 & -1.44 & 55.220 & 55.290 & -0.88 & 63.120 & 63.190 & -1.28 & 70.920 & 70.990 & -1.55 \\
\hline 47.320 & 48.460 & -1.46 & 55.320 & 55.390 & -0.52 & 63.220 & 63.290 & -1.09 & 71.020 & 71.090 & -1.61 \\
\hline 47.440 & 48.580 & -1.18 & 55.420 & 55.490 & -0.89 & 63.320 & 63.390 & -1.18 & 71.120 & 71.190 & -1.75 \\
\hline 47.520 & 48.660 & -1.50 & 55.520 & 55.590 & -0.98 & 63.420 & 63.490 & -1.22 & 71.320 & 71.390 & -1.70 \\
\hline 47.620 & 48.760 & -1.41 & 55.620 & 55.690 & -1.20 & 63.520 & 63.590 & -1.06 & 71.420 & 71.490 & -1.31 \\
\hline 47.720 & 48.860 & -1.74 & 55.720 & 55.790 & -1.25 & 63.620 & 63.690 & -1.32 & 71.520 & 71.590 & -1.47 \\
\hline 47.840 & 48.980 & -0.83 & 55.820 & 55.890 & -1.30 & 63.720 & 63.790 & -1.49 & 71.620 & 71.690 & -1.52 \\
\hline 47.920 & 49.060 & -1.18 & 55.920 & 55.990 & -1.24 & 63.790 & 63.860 & -1.45 & 71.720 & 71.790 & -1.17 \\
\hline 48.020 & 49.160 & -1.18 & 56.020 & 56.090 & -1.51 & 63.820 & 63.890 & -1.23 & 71.820 & 71.890 & -1.38 \\
\hline 48.140 & 49.280 & -1.13 & 56.120 & 56.190 & -1.30 & 63.920 & 63.990 & -1.03 & 71.920 & 71.990 & -1.39 \\
\hline 48.250 & 49.390 & -1.22 & 56.220 & 56.290 & -1.32 & 64.020 & 64.090 & -0.99 & 72.020 & 72.090 & -1.55 \\
\hline 48.330 & 49.470 & -1.42 & 56.320 & 56.390 & -1.13 & 64.120 & 64.190 & -0.97 & 72.120 & 72.190 & -1.42 \\
\hline 48.420 & 49.560 & -1.40 & 56.420 & 56.490 & -1.51 & 64.220 & 64.290 & -1.60 & 72.220 & 72.290 & -1.37 \\
\hline 48.510 & 49.650 & -1.68 & 56.520 & 56.590 & -1.00 & 64.320 & 64.390 & -1.23 & 72.320 & 72.390 & -1.56 \\
\hline 48.630 & 49.770 & -1.24 & 56.620 & 56.690 & -1.15 & 64.420 & 64.490 & -1.03 & 72.420 & 72.490 & -1.33 \\
\hline 48.730 & 49.870 & -1.15 & 56.720 & 56.790 & -1.60 & 64.520 & 64.590 & -1.12 & 72.520 & 72.590 & -1.39 \\
\hline 48.820 & 49.960 & -1.24 & 56.820 & 56.890 & -1.55 & 64.620 & 64.690 & -1.60 & 72.620 & 72.690 & -1.33 \\
\hline 48.940 & 50.080 & -1.13 & 57.020 & 57.090 & -1.36 & 64.720 & 64.790 & -1.56 & 72.720 & 72.780 & -1.35 \\
\hline 49.020 & 50.160 & -1.15 & 57.120 & 57.190 & -1.38 & 64.820 & 64.890 & -1.53 & 72.820 & 72.866 & -1.42 \\
\hline 49.120 & 50.260 & -1.18 & 57.220 & 57.290 & -1.38 & 64.920 & 64.990 & -1.51 & 72.920 & 72.953 & -1.70 \\
\hline 49.220 & 50.360 & -1.16 & 57.320 & 57.390 & -0.89 & 65.020 & 65.090 & -1.55 & 73.020 & 73.039 & -1.75 \\
\hline 49.340 & 50.480 & -1.14 & 57.420 & 57.490 & -1.05 & 65.120 & 65.190 & -1.39 & 73.020 & 73.039 & -1.54 \\
\hline 49.420 & 50.560 & -1.18 & 57.520 & 57.590 & -1.22 & 65.220 & 65.290 & -1.48 & 73.120 & 73.125 & -1.49 \\
\hline 49.520 & 50.707 & -1.24 & 57.620 & 57.690 & -1.20 & 65.320 & 65.390 & -1.61 & 73.140 & 73.142 & -1.67 \\
\hline 49.640 & 50.747 & -1.25 & 57.720 & 57.790 & -1.70 & 65.420 & 65.490 & -1.33 & 73.220 & 73.211 & -1.38 \\
\hline 49.750 & 50.785 & -1.51 & 57.820 & 57.890 & -1.60 & 65.520 & 65.590 & -1.45 & 73.240 & 73.228 & -1.54 \\
\hline 49.830 & 50.812 & -0.91 & 57.920 & 57.990 & -1.89 & 65.720 & 65.790 & -1.25 & 73.320 & 73.297 & -1.47 \\
\hline 49.920 & 50.842 & -1.03 & 58.020 & 58.090 & -1.87 & 65.820 & 65.890 & -1.63 & 73.420 & 73.383 & -1.63 \\
\hline 50.010 & 50.873 & -1.04 & 58.120 & 58.190 & -1.57 & 65.920 & 65.990 & -1.62 & 73.520 & 73.469 & -1.60 \\
\hline 50.130 & 50.914 & -1.15 & 58.220 & 58.290 & -1.31 & 66.020 & 66.090 & -1.36 & 73.620 & 73.555 & -1.37 \\
\hline 50.520 & 51.046 & -1.24 & 58.320 & 58.390 & -1.40 & 66.120 & 66.190 & -1.28 & 73.720 & 73.642 & -1.68 \\
\hline 50.620 & 51.080 & -1.17 & 58.420 & 58.490 & -1.34 & 66.220 & 66.290 & -1.52 & 73.820 & 73.728 & -1.64 \\
\hline 50.720 & 51.113 & -1.06 & 58.520 & 58.590 & -1.23 & 66.320 & 66.390 & -1.10 & 73.920 & 73.814 & -1.65 \\
\hline 50.840 & 51.154 & -1.28 & 58.620 & 58.690 & -1.52 & 66.420 & 66.490 & -1.60 & 74.020 & 73.900 & -1.37 \\
\hline 50.920 & 51.181 & -1.32 & 58.720 & 58.790 & -1.50 & 66.520 & 66.590 & -1.65 & 74.120 & 73.990 & -1.57 \\
\hline 51.020 & 51.215 & -1.50 & 58.820 & 58.890 & -1.35 & 66.620 & 66.690 & -1.39 & 74.220 & 74.077 & -1.45 \\
\hline 51.140 & 51.256 & -1.29 & 58.920 & 58.990 & -1.62 & 66.720 & 66.790 & -1.44 & 74.320 & 74.151 & -1.48 \\
\hline 51.250 & 51.293 & -1.30 & 59.020 & 59.090 & -1.50 & 66.820 & 66.890 & -1.41 & 74.420 & 74.225 & -1.43 \\
\hline 51.330 & 51.345 & -1.13 & 59.120 & 59.190 & -1.63 & 66.920 & 66.990 & -1.71 & 74.520 & 74.298 & -1.50 \\
\hline 51.420 & 51.449 & -1.15 & 59.220 & 59.290 & -1.57 & 67.020 & 67.090 & -1.50 & 74.620 & 74.380 & -1.43 \\
\hline 51.510 & 51.554 & -1.20 & 59.320 & 59.390 & -1.51 & 67.120 & 67.190 & -1.51 & 74.820 & 74.580 & -1.36 \\
\hline 51.630 & 51.693 & -1.38 & 59.420 & 59.490 & -1.76 & 67.220 & 67.290 & -1.61 & 74.920 & 74.680 & -1.46 \\
\hline 51.730 & 51.808 & -1.34 & 59.520 & 59.590 & -1.57 & 67.320 & 67.390 & -1.25 & 75.020 & 74.780 & -1.67 \\
\hline 51.820 & 51.913 & -1.44 & 59.620 & 59.690 & -1.22 & 67.420 & 67.490 & -1.39 & 75.120 & 74.880 & -1.62 \\
\hline 51.940 & 52.052 & -1.42 & 59.720 & 59.790 & -1.33 & 67.520 & 67.590 & -1.25 & 75.220 & 74.980 & -1.53 \\
\hline 52.020 & 52.145 & -1.34 & 59.820 & 59.890 & -1.16 & 67.620 & 67.690 & -1.31 & 75.320 & 75.080 & -1.50 \\
\hline 52.120 & 52.261 & -1.31 & 59.920 & 59.990 & -1.46 & 67.720 & 67.790 & -1.47 & 75.420 & 75.180 & -1.24 \\
\hline 52.220 & 52.377 & -1.62 & 60.020 & 60.090 & -1.35 & 67.820 & 67.890 & -1.73 & 75.520 & 75.280 & -1.35 \\
\hline 52.340 & 52.516 & -0.94 & 60.120 & 60.190 & -1.24 & 67.920 & 67.990 & -1.41 & 75.620 & 75.380 & -1.36 \\
\hline 52.420 & 52.609 & -1.08 & 60.220 & 60.290 & -1.11 & 68.020 & 68.090 & -1.45 & 75.720 & 75.480 & -1.35 \\
\hline 52.520 & 52.724 & -1.28 & 60.320 & 60.390 & -1.22 & 68.120 & 68.190 & -1.33 & 75.820 & 75.580 & -1.54 \\
\hline
\end{tabular}


Table 1 (continued).

\begin{tabular}{|c|c|c|c|c|c|c|c|c|c|c|c|}
\hline $\begin{array}{l}\text { Depth } \\
\text { (mbsf) }\end{array}$ & $\begin{array}{l}\text { Splice depth (m) } \\
\text { (MCD) }\end{array}$ & $\delta^{18} \mathrm{O}$ & $\begin{array}{l}\text { Depth } \\
\text { (mbsf) }\end{array}$ & $\begin{array}{l}\text { Splice depth }(\mathrm{m}) \\
\text { (MCD) }\end{array}$ & $\delta^{18} \mathrm{O}$ & $\begin{array}{l}\text { Depth } \\
\text { (mbsf) }\end{array}$ & $\begin{array}{l}\text { Splice depth }(\mathrm{m}) \\
\text { (MCD) }\end{array}$ & $\delta^{18} \mathrm{O}$ & $\begin{array}{l}\text { Depth } \\
\text { (mbsf) }\end{array}$ & $\begin{array}{l}\text { Splice depth (m) } \\
\text { (MCD) }\end{array}$ & $\delta^{18} \mathrm{O}$ \\
\hline 75.920 & 75.680 & -1.40 & 84.020 & 84.440 & -1.37 & 92.110 & 92.530 & -1.41 & 100.22 & 100.64 & -1.52 \\
\hline 76.020 & 75.780 & -1.46 & 84.120 & 84.540 & -1.40 & 92.150 & 92.570 & -1.82 & 100.32 & 100.74 & -1.77 \\
\hline 76.120 & 75.880 & -1.47 & 84.220 & 84.640 & -1.38 & 92.250 & 92.670 & -1.92 & 100.52 & 100.94 & -1.94 \\
\hline 76.220 & 75.980 & -1.46 & 84.320 & 84.740 & -1.43 & 92.520 & 92.940 & -1.45 & 100.82 & 101.24 & -1.54 \\
\hline 76.320 & 76.080 & -1.47 & 84.420 & 84.840 & -1.39 & 92.620 & 93.040 & -1.74 & 100.92 & 101.34 & -1.73 \\
\hline 76.420 & 76.180 & -1.43 & 84.520 & 84.940 & -1.51 & 92.720 & 93.140 & -1.71 & 101.72 & 102.14 & -1.42 \\
\hline 76.520 & 76.280 & -1.36 & 84.620 & 85.040 & -1.73 & 92.820 & 93.240 & -1.56 & 101.82 & 102.24 & -1.57 \\
\hline 76.620 & 76.380 & -1.31 & 84.720 & 85,140 & -1.29 & 92.920 & 93.340 & -1.64 & 101.88 & 102.30 & -1.56 \\
\hline 76.720 & 76.480 & -1.24 & 84.820 & 85.240 & -1.50 & 93.020 & 93.440 & -1.54 & 101.92 & 102.34 & -1.59 \\
\hline 76.820 & 76.580 & -1.15 & 84.920 & 85.340 & -1.55 & 93.120 & 93.540 & -1.55 & 101.97 & 102.39 & -1.62 \\
\hline 76.920 & 76.680 & -1.13 & 85.020 & 85.440 & -1.54 & 93.220 & 93.640 & -1.63 & 102.02 & 102.44 & -1.63 \\
\hline 77.020 & 76.780 & -1.26 & 85.120 & 85.540 & -1.49 & 93.320 & 93.740 & -1.73 & 102.12 & 102.54 & -1.69 \\
\hline 77.120 & 76.880 & -1.17 & 85.220 & 85.640 & -1.54 & 93.420 & 93.840 & -1.51 & 102.22 & 102.64 & -1.70 \\
\hline 77.220 & 76.980 & -1.46 & 85.320 & 85.740 & -1.39 & 93.530 & 93.950 & -1.32 & 102.32 & 102.74 & -1.67 \\
\hline 77.320 & 77.080 & -1.18 & 85.420 & 85.840 & -1.53 & 93.620 & 94.040 & -1.59 & 102.42 & 102.84 & -1.51 \\
\hline 77.420 & 77.180 & -1.24 & 85.520 & 85.940 & -1.42 & 93.720 & 94.140 & -1.55 & 102.52 & 102.94 & -1.68 \\
\hline 77.520 & 77.278 & -1.39 & 85.620 & 86.040 & -1.47 & 93.820 & 94.240 & -1.50 & 102.63 & 103.05 & -1.49 \\
\hline 77.620 & 77.405 & -1.43 & 85.820 & 86.240 & -1.63 & 93.920 & 94.340 & -1.21 & 102.73 & 103.15 & -1.49 \\
\hline 77.720 & 77.532 & -1.43 & 85.920 & 86.340 & -1.43 & 94.020 & 94.440 & -1.50 & 102.92 & 103.34 & -1.54 \\
\hline 77.820 & 77.659 & -1.43 & 86.020 & 86.440 & -1.71 & 94.120 & 94.540 & -1.76 & 103.02 & 103.44 & -1.61 \\
\hline 77.920 & 77.786 & -1.50 & 86.120 & 86.540 & -1.53 & 94.220 & 94.640 & -1.65 & 103.12 & 103.54 & -1.66 \\
\hline 78.020 & 77.914 & -1.46 & 86.220 & 86.640 & -1.51 & 94.320 & 94.740 & -1.58 & 103.22 & 103.64 & -1.80 \\
\hline 78.120 & 78.041 & -1.41 & 86.320 & 86.740 & -1.45 & 94.420 & 94.840 & -1.53 & 103.32 & 103.74 & -1.77 \\
\hline 78.220 & 78.168 & -1.55 & 86.420 & 86.840 & -1.64 & 94.520 & 94.940 & $-1,44$ & 103.42 & 103.84 & -1.54 \\
\hline 78.320 & 78.295 & -1.40 & 86.520 & 86.940 & -1.67 & 94.620 & 95.040 & -1.33 & 103.52 & 103.94 & -1.52 \\
\hline 78.420 & 78.422 & -1.34 & 86.620 & 87.040 & -1.50 & 94.720 & 95.140 & -1.68 & 103.62 & 104.04 & -1.32 \\
\hline 78.520 & 78.549 & -1.38 & 86.720 & 87.140 & -1.41 & 94.820 & 95.240 & -1.50 & 103.72 & 104.14 & -1.53 \\
\hline 78.620 & 78.676 & -1.37 & 86.820 & 87.240 & -1.45 & 94.920 & 95.340 & -1.42 & 103.82 & 104.24 & -1.61 \\
\hline 79.020 & 79.185 & -1.52 & 86.920 & 87.340 & -1.64 & 95.030 & 95.450 & -1.57 & 103.92 & 104.34 & -1.51 \\
\hline 79.120 & 79.312 & -1.46 & 87.020 & 87.440 & -1.46 & 95.120 & 95.540 & -1.51 & 104.02 & 104.44 & -1.69 \\
\hline 79.220 & 79.439 & -1.42 & 87.120 & 87.540 & -1.57 & 95.220 & 95.640 & -1.46 & 104.12 & 104.54 & -1.54 \\
\hline 79.320 & 79.566 & -1.33 & 87.220 & 87.640 & -1.66 & 95.320 & 95.740 & -1.47 & 104.23 & 104.65 & -1.34 \\
\hline 79.420 & 79.693 & -1.28 & 87.320 & 87.740 & -1.52 & 95.520 & 95.940 & -1.63 & 104.32 & 104.74 & -1.61 \\
\hline 79.520 & 79.820 & -1.17 & 87.420 & 87.840 & -1.54 & 95.620 & 96.040 & -1.61 & 104.42 & 104.84 & -1.48 \\
\hline 79.620 & 79.947 & -1.25 & 87.520 & 87.940 & -1.43 & 95.720 & 96.140 & -1.63 & 104.52 & 104.94 & -1.65 \\
\hline 79.720 & 80.074 & -1.32 & 87.620 & 88.040 & -1.41 & 95.820 & 96.240 & -1.85 & 104.62 & 105.04 & -1.64 \\
\hline 79.820 & 80.201 & -1.48 & 87.720 & 88.140 & -1.49 & 95.920 & 96.340 & -1.63 & 104.72 & 105.14 & -1.58 \\
\hline 79.920 & 80.328 & -1.36 & 87.820 & 88.240 & -1.76 & 96.020 & 96.440 & -1.23 & 104.82 & 105.24 & -1.59 \\
\hline 80.020 & 80.440 & -1.36 & 87.920 & 88.340 & -1.34 & 96.120 & 96.540 & -1.59 & 104.92 & 105.34 & -1.52 \\
\hline 80.120 & 80.540 & -1.51 & 88.020 & 88.440 & -1.58 & 96.220 & 96.640 & -1.88 & 105.02 & 105.44 & -1.53 \\
\hline 80.220 & 80.640 & -1.58 & 88.120 & 88.540 & -1.49 & 96.320 & 96.740 & -1.85 & 105.12 & 105.54 & -1.52 \\
\hline 80.320 & 80.740 & -1.52 & 88.220 & 88.640 & -1.55 & 96.420 & 96.840 & -1.37 & 105.32 & 105.74 & -1.68 \\
\hline 80.420 & 80.840 & -1.36 & 88.320 & 88.740 & -1.44 & 96.530 & 96.950 & -1.80 & 105.42 & 105.84 & -1.54 \\
\hline 80.520 & 80.940 & -1.63 & 88.420 & 88.840 & -1.54 & 96.620 & 97.040 & -1.94 & 105.52 & 105.94 & -1.73 \\
\hline 80.620 & 81.040 & -1.46 & 88.520 & 88.940 & -1.47 & 96.720 & 97.140 & -1.64 & 105.62 & 106.04 & -1.68 \\
\hline 80.720 & 81.140 & -1.65 & 88.620 & 89.040 & -1.31 & 96.820 & 97.240 & -1.39 & 105.72 & 106.14 & -1.66 \\
\hline 80.820 & 81.240 & -1.43 & 88.720 & 89.140 & -1.66 & 96.920 & 97.340 & -1.67 & 105.73 & 106.15 & -1.54 \\
\hline 80.920 & 81.340 & -1.46 & 88.820 & 89.240 & -1.32 & 97.020 & 97.440 & -1.54 & 105.82 & 106.24 & -1.67 \\
\hline 81.020 & 81.440 & -1.67 & 88.920 & 89.340 & -1.27 & 97.120 & 97.540 & -1.46 & 105.92 & 106.34 & -1.62 \\
\hline 81.120 & 81.540 & -1.81 & 89.020 & 89.440 & -1.27 & 97.220 & 97.640 & -1.62 & 106.02 & 106.44 & -1.70 \\
\hline 81.220 & 81.640 & -1.77 & 89.120 & 89.540 & -1.52 & 97.320 & 97.740 & -1.46 & 106.12 & 106.54 & -1.65 \\
\hline 81.320 & 81.740 & -1.65 & 89.220 & 89.640 & -1.38 & 97.420 & 97.840 & -1.57 & 106.22 & 106.64 & -1.50 \\
\hline 81.420 & 81.840 & -1.78 & 89.320 & 89.740 & -1.49 & 97.520 & 97.940 & -1.64 & 106.31 & 106.73 & -1.43 \\
\hline 81.520 & 81.940 & -1.65 & 89.420 & 89.840 & -1.47 & 97.620 & 98.040 & -1.62 & 106.42 & 106.84 & -1.28 \\
\hline 81.620 & 82.040 & -1.54 & 89.520 & 89.940 & -1.54 & 97.720 & 98.140 & -1.29 & 106.52 & 106.94 & -1.48 \\
\hline 81.720 & 82.140 & -1.47 & 89.620 & 90.040 & -1.45 & 97.820 & 98.240 & -1.62 & 106.62 & 107.04 & -1.65 \\
\hline 81.820 & 82.240 & -1.44 & 89.720 & 90.140 & -1.73 & 97.920 & 98.340 & -1.72 & 106.72 & 107.14 & -1.54 \\
\hline 81.920 & 82.340 & -1.54 & 89.820 & 90.240 & -1.65 & 98.030 & 98.450 & -1.55 & 106.82 & 107.24 & -1.62 \\
\hline 82.020 & 82.440 & -1.57 & 89.920 & 90.340 & -1.65 & 98.120 & 98.540 & -1.46 & 106.92 & 107.34 & -1.55 \\
\hline 82.120 & 82.540 & -1.79 & 90.020 & 90.440 & -1.49 & 98.220 & 98.640 & -1.78 & 107.02 & 107.44 & -1.62 \\
\hline 82.220 & 82.640 & -1.76 & 90.120 & 90.540 & -1.62 & 98.320 & 98.740 & -1.72 & 107.12 & 107.54 & -1.56 \\
\hline 82.320 & 82.740 & -1.46 & 90.220 & 90.640 & -1.56 & 98.420 & 98.840 & -1.61 & 107.52 & 107.94 & -1.31 \\
\hline 82.420 & 82.840 & -1.53 & 90.420 & 90.840 & -1.54 & 98.520 & 98.940 & -1.54 & 107.62 & 108.04 & -1.48 \\
\hline 82.520 & 82.940 & -1.41 & 90.520 & 90.940 & -1.56 & 98.620 & 99.040 & -1.70 & 107.72 & 108.14 & -1.42 \\
\hline 82.620 & 83.040 & -1.49 & 90.620 & 91.040 & -1.62 & 98.720 & 99.140 & -1.72 & 107.82 & 108.24 & -1.62 \\
\hline 82.720 & 83.140 & -1.38 & 90.720 & 91.140 & -1.70 & 98.820 & 99.240 & -1.39 & 107.92 & 108.34 & -1.38 \\
\hline 82.820 & 83.240 & -1.57 & 90.820 & 91.240 & -1.51 & 98.920 & 99.340 & -1.78 & 108.02 & 108.44 & -1.38 \\
\hline 82.910 & 83.330 & -1.41 & 90.920 & 91.340 & -1.68 & 99.020 & 99.440 & -1.56 & 108.12 & 108.54 & -1.52 \\
\hline 83.020 & 83.440 & -1.50 & 91.020 & 91.440 & -1.62 & 99.150 & 99.570 & -1.67 & 108.22 & 108.64 & -1.36 \\
\hline 83.120 & 83.540 & -1.52 & 91.120 & 91.540 & -1.47 & 99.220 & 99.640 & -1.75 & 108.32 & 108.74 & -1.37 \\
\hline 83.220 & 83.640 & -1.53 & 91.220 & 91.640 & -1.65 & 99.320 & 99.740 & -1.56 & 108.42 & 108.84 & -1.23 \\
\hline 83.320 & 83.740 & -1.43 & 91.320 & 91.740 & -1.52 & 99.420 & 99.840 & -1.62 & 108.52 & 108.94 & -1.37 \\
\hline 83.420 & 83.840 & -1.47 & 91.420 & 91.840 & -1.32 & 99.530 & 99.950 & -1.63 & 108.64 & 109.06 & -1.56 \\
\hline 83.520 & 83.940 & -1.50 & 91.520 & 91.940 & -1.48 & 99.620 & 100.04 & -1.69 & 108.73 & 109.15 & -1.51 \\
\hline 83.620 & 84.040 & -1.73 & 91.620 & 92.040 & -1.48 & 99.820 & 100.24 & -1.43 & 108.82 & 109.24 & -1.61 \\
\hline 83.720 & 84.140 & -1.29 & 91.720 & 92.140 & -1.57 & 99.920 & 100.34 & -1.70 & 108.92 & 109.34 & -1.42 \\
\hline 83.820 & 84.240 & -1.56 & 91.920 & 92.340 & -1.57 & 100.02 & 100.44 & -1.25 & 109.02 & 109.44 & -1.09 \\
\hline 83.920 & 84.340 & -1.63 & 92.020 & 92.440 & -1.60 & 100.12 & 100.54 & -1.36 & 109.12 & 109.54 & -1.30 \\
\hline
\end{tabular}


Table 1 (continued).

\begin{tabular}{|c|c|c|c|c|c|c|c|c|c|c|c|}
\hline $\begin{array}{l}\text { Depth } \\
\text { (mbsf) }\end{array}$ & $\begin{array}{l}\text { Splice depth (m) } \\
\text { (MCD) }\end{array}$ & $\delta^{18} \mathrm{O}$ & $\begin{array}{l}\text { Depth } \\
\text { (mbsf) }\end{array}$ & $\begin{array}{l}\text { Splice depth (m) } \\
\text { (MCD) }\end{array}$ & $\delta^{18} \mathrm{O}$ & $\begin{array}{l}\text { Depth } \\
\text { (mbsf) }\end{array}$ & $\begin{array}{l}\text { Splice depth (m) } \\
\text { (MCD) }\end{array}$ & $\delta^{18} \mathrm{O}$ & $\begin{array}{l}\text { Depth } \\
\text { (mbsf) }\end{array}$ & $\begin{array}{l}\text { Splice depth }(\mathrm{m}) \\
(\mathrm{MCD})\end{array}$ & $\delta^{18} \mathrm{O}$ \\
\hline 109.33 & 109.75 & -1.43 & 117.32 & 117.74 & -1.59 & 126.52 & 126.94 & -1.09 & 134.82 & 135.24 & -1.58 \\
\hline 109.42 & 109.84 & -1.37 & 117.42 & 117.84 & -1.48 & 126.62 & 127.04 & -1.26 & 134.92 & 135.34 & -1.15 \\
\hline 109.52 & 109.94 & -1.35 & 117.52 & 117.94 & -1.51 & 126.72 & 127.14 & -1.38 & 135.02 & 135.44 & -1.28 \\
\hline 109.62 & 110.04 & -1.36 & 117.72 & 118.14 & -1.53 & 126.80 & 127.22 & -1.37 & 135.12 & 135.54 & -1.47 \\
\hline 109.72 & 110.14 & -1.41 & 117.82 & 118.24 & -1.54 & 126.92 & 127.34 & -1.31 & 135.22 & 135.64 & -1.18 \\
\hline 109.82 & 110.24 & -1.45 & 117.92 & 118.34 & -1.33 & 127.02 & 127.44 & -1.43 & 135.32 & 135.74 & -1.25 \\
\hline 109.92 & 110.34 & -1.56 & 118.02 & 118.44 & -1.39 & 127.12 & 127.54 & -0.81 & 135.42 & 135.84 & -1.56 \\
\hline 110.02 & 110.44 & -1.57 & 118.12 & 118.54 & -1.36 & 127.22 & 127.64 & -1.09 & 135.52 & 135.94 & -1.36 \\
\hline 110.12 & 110.54 & -1.64 & 118.23 & 118.65 & -1.63 & 127.32 & 127.74 & -1.22 & 135.62 & 136.04 & -0.74 \\
\hline 110.23 & 110.65 & -1.43 & 118.32 & 118.74 & -1.49 & 127.42 & 127.84 & -1.34 & 136.02 & 136.44 & -1.35 \\
\hline 110.32 & 110.74 & -1.68 & 118.52 & 118.94 & -1.28 & 127.52 & 127.94 & -1.38 & 136.12 & 136.54 & -1.52 \\
\hline 110.42 & 110.84 & -1.66 & 118.62 & 119.04 & -1.48 & 127.73 & 128.15 & -1.37 & 136.22 & 136.64 & -1.32 \\
\hline 110.52 & 110.94 & -1.59 & 118.72 & 119.14 & -1.37 & 127.82 & 128.24 & -1.03 & 136.32 & 136.74 & -1.48 \\
\hline 110.62 & 111.04 & -1.64 & 118.82 & 119.24 & -1.34 & 127.92 & 128.34 & -1.16 & 136.42 & 136.84 & -1.33 \\
\hline 110.72 & 111.14 & -1.57 & 118.92 & 119.34 & -1.61 & 128.02 & 128.44 & -1.10 & 136.52 & 136.94 & -1.42 \\
\hline 110.82 & 111.24 & -1.44 & 119.02 & 119.44 & -1.47 & 128.12 & 128.54 & -1.34 & 136.62 & 137.04 & -1.51 \\
\hline 110.92 & 111.34 & -1.28 & 119.12 & 119.54 & -1.48 & 128.22 & 128.64 & -1.25 & 136.72 & 137.14 & -1.41 \\
\hline 111.02 & 111.44 & -1.57 & 119.22 & 119.64 & -1.41 & 128.32 & 128.74 & -1.34 & 136.82 & 137.24 & -1.31 \\
\hline 111.12 & 111.54 & -1.42 & 119.42 & 119.84 & -1.33 & 128.42 & 128.84 & -1.43 & 136.92 & 137.34 & -1.32 \\
\hline 111.22 & 111.64 & -1.49 & 119.52 & 119.94 & -1.58 & 128.52 & 128.94 & -1.52 & 137.02 & 137.44 & -1.26 \\
\hline 111.29 & 111.71 & -1.77 & 119.62 & 120.04 & -1.21 & 128.62 & 129.04 & -1.44 & 137.12 & 137.54 & -1.37 \\
\hline 111.32 & 111.74 & -1.37 & 119.73 & 120.15 & -1.24 & 128.72 & 129.14 & -1.36 & 137.23 & 137.65 & -1.45 \\
\hline 111.42 & 111.84 & -1.56 & 119.82 & 120.24 & -1.52 & 128.82 & 129.24 & -1.28 & 137.32 & 137.74 & -1.49 \\
\hline 111.52 & 111.94 & -1.28 & 119.92 & 120.34 & -1.55 & 128.92 & 129.34 & -1.40 & 137.42 & 137.84 & -1.53 \\
\hline 111.62 & 112.04 & -1.47 & 120.02 & 120.44 & -1.43 & 129.02 & 129.44 & -1.34 & 137.52 & 137.94 & -1.16 \\
\hline 111.72 & 112.14 & -1.51 & 120.12 & 120.54 & -1.55 & 129.23 & 129.65 & -1.40 & 137.62 & 138.04 & -0.97 \\
\hline 111.82 & 112.24 & -1.45 & 120.22 & 120.64 & -1.43 & 129.32 & 129.74 & -1.48 & 137.72 & 138.14 & -1.16 \\
\hline 111.92 & 112.34 & -1.41 & 120.32 & 120.74 & -1.39 & 129.42 & 129.84 & -1.30 & 137.82 & 138.24 & -1.20 \\
\hline 112.02 & 112.44 & -1.40 & 120.82 & 121.24 & -1.49 & 129.52 & 129.94 & -1.31 & 137.92 & 138.34 & -1.39 \\
\hline 112.12 & 112.54 & -1.48 & 120.84 & 121.26 & -1.35 & 129.62 & 130.04 & -1.33 & 138.02 & 138.44 & -1.56 \\
\hline 112.23 & 112.65 & -1.54 & 120.92 & 121.34 & -1.34 & 129.68 & 130.10 & -1.44 & 138.12 & 138.54 & -1.60 \\
\hline 112.32 & 112.74 & -1.54 & 121.02 & 121.44 & -1.39 & 129.82 & 130.24 & -1.30 & 138.22 & 138.64 & -1.76 \\
\hline 112.42 & 112.84 & -1.58 & 121.12 & 121.54 & -1.57 & 129.92 & 130.34 & -1.34 & 138.32 & 138.74 & -1.64 \\
\hline 112.52 & 112.94 & -1.55 & 121.32 & 121.74 & -1.38 & 130.02 & 130,44 & -1.17 & 138.42 & 138.84 & -1.16 \\
\hline 112.62 & 113.04 & -1.31 & 121.42 & 121.84 & -1.55 & 130.12 & 130.54 & -1.20 & 138.52 & 138.94 & -1.26 \\
\hline 112.72 & 113.14 & -1.84 & 121.73 & 122.15 & -1.17 & 130.32 & 130.74 & -0.96 & 138.62 & 139.04 & -1.46 \\
\hline 112.82 & 113.24 & -1.50 & 121.82 & 122.24 & -1.12 & 130.42 & 130.84 & -1.17 & 138.73 & 139.15 & -1.37 \\
\hline 112.92 & 113.34 & -1.56 & 121.92 & 122.34 & -0.91 & 130.52 & 130.94 & -1.16 & 138.82 & 139.24 & -1.25 \\
\hline 113.04 & 113.46 & -1.49 & 122.02 & 122.44 & -1.17 & 130.62 & 131.04 & -1.10 & 138.92 & 139.34 & -1.35 \\
\hline 113.12 & 113.54 & -1.55 & 122.12 & 122.54 & -1.32 & 130.82 & 131.24 & -1.23 & 139.02 & 139.44 & -1.16 \\
\hline 113.22 & 113.64 & -1.37 & 122.22 & 122.64 & -1.23 & 130.92 & 131.34 & -1.14 & 139.12 & 139.54 & -1.02 \\
\hline 113.32 & 113.74 & -1.29 & 122.32 & 122.74 & -1.10 & 131.02 & 131.44 & -1.11 & 139.22 & 139.64 & -1.46 \\
\hline 113.42 & 113.84 & -1.13 & 122.42 & 122.84 & -1.35 & 131.12 & 131.54 & -0.97 & 139.32 & 139.74 & -1.85 \\
\hline 113.52 & 113.94 & -1.38 & 122.52 & 122.94 & -1.51 & 131.23 & 131.65 & -1.31 & 139.42 & 139.84 & -1.44 \\
\hline 113.62 & 114.04 & -1.38 & 122.62 & 123.04 & -1.41 & 131.32 & 131.74 & -1.34 & 139.52 & 139.94 & -1.37 \\
\hline 113.73 & 114.15 & -1.51 & 122.72 & 123.14 & -1.44 & 131.42 & 131.84 & -1.17 & 139.62 & 140.04 & -1.40 \\
\hline 113.82 & 114.24 & -1.56 & 122.82 & 123.24 & -1.40 & 131.52 & 131.94 & -1.27 & 139.72 & 140.14 & -1.33 \\
\hline 113.92 & 114.34 & -1.68 & 122.92 & 123.34 & -1.43 & 131.62 & 132.04 & -1.20 & 139.82 & 140.24 & -1.10 \\
\hline 114.02 & 114.44 & -1.58 & 123.02 & 123.44 & -1.41 & 131.72 & 132.14 & -1.19 & 139.92 & 140.34 & -1.37 \\
\hline 114.12 & 114.54 & -1.51 & 123.23 & 123.65 & -1.32 & 131.82 & 132.24 & -1.06 & 140.02 & 140.44 & -1.30 \\
\hline 114.22 & 114.64 & -1.33 & 123.32 & 123.74 & -1.69 & 131.92 & 132.34 & -1.15 & 140.12 & 140.54 & -1.31 \\
\hline 114.31 & 114.73 & -1.35 & 123.42 & 123.84 & -1.42 & 132.02 & 132.44 & -1.30 & 140.22 & 140.64 & -1.15 \\
\hline 114.42 & 114.84 & -1.35 & 123.52 & 123.94 & -1.26 & 132.12 & 132.54 & -1.04 & 140.32 & 140.74 & -1.32 \\
\hline 114.51 & 114.93 & -1.29 & 123.62 & 124.04 & -1.22 & 132.22 & 132.64 & -1.27 & 140.42 & 140.84 & -1.24 \\
\hline 114.62 & 115.04 & -1.59 & 123.72 & 124.14 & -1.26 & 132.32 & 132.74 & -1.14 & 140.52 & 140.94 & -1.56 \\
\hline 115.02 & 115.44 & -1.51 & 123.82 & 124.24 & -1.20 & 132.42 & 132.84 & -1.18 & 140.62 & 141.04 & -1.45 \\
\hline 115.12 & 115.54 & -1.40 & 123.92 & 124.34 & -1.32 & 132.52 & 132.94 & -1.05 & 140.73 & 141.15 & -1.43 \\
\hline 115.23 & 115.65 & -1.48 & 124.02 & 124.44 & -1.27 & 132.62 & 133.04 & -1.26 & 140.82 & 141.24 & -1.52 \\
\hline 115.32 & 115.74 & -1.48 & 124.12 & 124.54 & -1.21 & 132.73 & 133.15 & -1.27 & 140.92 & 141.34 & -1.32 \\
\hline 115.42 & 115.84 & -1.37 & 124.22 & 124.64 & -1.25 & 132.82 & 133.24 & -1.21 & 141.02 & 141.44 & -1.56 \\
\hline 115.52 & 115.94 & -1.53 & 124.32 & 124.74 & -1.26 & 132.92 & 133.34 & -1.32 & 141.12 & 141.54 & -1.71 \\
\hline 115.62 & 116.04 & -1.49 & 124.42 & 124.84 & -1.28 & 133.02 & 133.44 & -1.22 & 141.22 & 141.64 & -1.49 \\
\hline 115.72 & 116.14 & -1.35 & 124.52 & 124.94 & -1.57 & 133.12 & 133.54 & -1.30 & 141.32 & 141.74 & -1.35 \\
\hline 115.81 & 116.23 & -1.41 & 124.73 & 125.15 & -1.55 & 133.22 & 133.64 & -1.39 & 141.52 & 141.94 & -1.22 \\
\hline 115.92 & 116.34 & -1.53 & 124.81 & 125.23 & -1.24 & 133.32 & 133.74 & -1.16 & 141.62 & 142.04 & -1.12 \\
\hline 116.01 & 116.43 & -1.33 & 124.92 & 125.34 & -1.47 & 133.42 & 133.84 & -1.28 & 141.72 & 142.14 & -1.08 \\
\hline 116.12 & 116.54 & -1.37 & 125.02 & 125.44 & -1.26 & 133.52 & 133.94 & -1.23 & 141.82 & 142.24 & -1.16 \\
\hline 116.22 & 116.64 & -1.36 & 125.12 & 125.54 & -1.50 & 133.62 & 134.04 & -0.74 & 141.92 & 142.34 & -1.14 \\
\hline 116.32 & 116.74 & -1.50 & 125.22 & 125.64 & -1.68 & 133.82 & 134.24 & -1.24 & 142.02 & 142.44 & -1.22 \\
\hline 116.42 & 116.84 & -1.51 & 125.30 & 125.72 & -1.26 & 133.92 & 134.34 & -1.19 & 142.12 & 142.54 & -1.21 \\
\hline 116.52 & 116.94 & -1.59 & 125.42 & 125.84 & -1.31 & 134.02 & 134.44 & -1.40 & 142.23 & 142.65 & -1.23 \\
\hline 116.62 & 117.04 & -1.56 & 125.52 & 125.94 & -1.47 & 134.12 & 134.54 & -1.51 & 142.32 & 142.74 & -1.30 \\
\hline 116.73 & 117.15 & -1.58 & 125.62 & 126.04 & -1.28 & 134.23 & 134.65 & -1.26 & 142.42 & 142.84 & -1.34 \\
\hline 116.82 & 117.24 & -1.19 & 125.82 & 126.24 & -1.33 & 134.32 & 134.74 & -1.38 & 142.52 & 142.94 & -1.30 \\
\hline 116.92 & 117.34 & -1.46 & 125.92 & 126.34 & -1.37 & 134.42 & 134.84 & -1.10 & 142.62 & 143.04 & -1.74 \\
\hline 117.02 & 117.44 & -1.58 & 126.23 & 126.65 & -1.35 & 134.52 & 134.94 & -1.19 & 142.72 & 143.14 & -1.40 \\
\hline 117.12 & 117.54 & -1.62 & 126.32 & 126.74 & -1.20 & 134.62 & 135.04 & -1.07 & 142.82 & 143.24 & -1.61 \\
\hline 117.22 & 117.64 & -1.56 & 126.42 & 126.84 & -1.30 & 134.72 & 135.14 & -1.16 & 142.92 & 143.34 & -1.30 \\
\hline
\end{tabular}


Table 1 (continued).

\begin{tabular}{|c|c|c|c|c|c|}
\hline $\begin{array}{l}\text { Depth } \\
\text { (mbsf) }\end{array}$ & $\begin{array}{l}\text { Splice depth (m) } \\
\text { (MCD) }\end{array}$ & $\delta^{18} \mathrm{O}$ & $\begin{array}{l}\text { Depth } \\
\text { (mbsf) }\end{array}$ & $\begin{array}{l}\text { Splice depth (m) } \\
\text { (MCD) }\end{array}$ & $\delta^{18} \mathrm{O}$ \\
\hline 143.02 & 143.44 & -1.47 & 148.52 & 148.94 & -1.36 \\
\hline 143.12 & 143.54 & -1.39 & 148.62 & 149.04 & -1.49 \\
\hline 143.22 & 143.64 & -1.09 & 148.72 & 149.14 & -1.36 \\
\hline 143.32 & 143.74 & -1.58 & 148.82 & 149.24 & -1.31 \\
\hline 143.42 & 143.84 & -1.32 & 148.92 & 149.34 & -1.27 \\
\hline 143.52 & 143.94 & -1.40 & 149.02 & 149.44 & -1.49 \\
\hline 143.62 & 144.04 & -1.23 & 149.12 & 149.54 & -1.46 \\
\hline 143.73 & 144.15 & -1.24 & 149.22 & 149.64 & -1.65 \\
\hline 143.82 & 144.24 & -1.29 & 149.32 & 149.74 & -1.03 \\
\hline 143.92 & 144.34 & -1.44 & 149.42 & 149.84 & -1.05 \\
\hline 144.02 & 144.44 & -1.57 & 149.52 & 149.94 & -1.14 \\
\hline 144.12 & 144.54 & -1.30 & 149.62 & 150.04 & -1.07 \\
\hline 144.22 & 144.64 & -1.33 & 149.72 & 150.14 & -1.31 \\
\hline 144.32 & 144.74 & -1.34 & 149.82 & 150.24 & -1.19 \\
\hline 144.42 & 144.84 & -1.58 & 149.92 & 150.34 & -1.25 \\
\hline 144.52 & 144.94 & -1.45 & 150.02 & 150.44 & -1.25 \\
\hline 144.62 & 145.04 & -1.40 & 150.11 & 150.53 & -1.31 \\
\hline 144.82 & 145.24 & -1.26 & 150.24 & 150.66 & -1.13 \\
\hline 144.92 & 145.34 & -1.36 & 150.32 & 150.74 & -1.39 \\
\hline 145.02 & 145.44 & -1.41 & 150.42 & 150.84 & -1.36 \\
\hline 145.12 & 145.54 & -1.29 & 150.52 & 150.94 & -1.41 \\
\hline 145.23 & 145.65 & -0.98 & 150.62 & 151.04 & -1.05 \\
\hline 145.32 & 145.74 & -1.31 & 150.72 & 151.14 & -1.01 \\
\hline 145.42 & 145.84 & -1.31 & 150.82 & 151.24 & -1.36 \\
\hline 145.52 & 145.94 & -1.58 & 150.92 & 151.34 & -1.33 \\
\hline 145.62 & 146.04 & -1.79 & 151.02 & 151.44 & -1.25 \\
\hline 145.72 & 146.14 & -1.47 & 151.12 & 151.54 & -1.31 \\
\hline 145.82 & 146.24 & -1.43 & 151.22 & 151.64 & -1.44 \\
\hline 145.92 & 146.34 & -1.46 & 151.32 & 151.74 & -0.97 \\
\hline 146.02 & 146.44 & -1.46 & 151.42 & 151.84 & -1.10 \\
\hline 146.12 & 146.54 & -1.42 & 151.52 & 151.94 & -1.45 \\
\hline 146.22 & 146.64 & -1.32 & 151.62 & 152.04 & -1.27 \\
\hline 146.32 & 146.74 & -1.51 & 151.74 & 152.16 & -1.52 \\
\hline 146.42 & 146.84 & -1.24 & 151.82 & 152.24 & -1.03 \\
\hline 146.52 & 146.94 & -1.26 & 151.92 & 152.34 & -1.14 \\
\hline 146.62 & 147.04 & -1.35 & 152.02 & 152.44 & -1.37 \\
\hline 146.73 & 147.15 & -1.44 & 152.12 & 152.54 & -1.27 \\
\hline 146.82 & 147.24 & -1.32 & 152.22 & 152.64 & -1.34 \\
\hline 146.92 & 147.34 & -1.38 & 152.32 & 152.74 & -1.43 \\
\hline 147.02 & 147.44 & -1.27 & 152.42 & 152.84 & -1.42 \\
\hline 147.12 & 147.54 & -1.40 & 152.62 & 153.04 & -1.20 \\
\hline 147.32 & 147.74 & -1.65 & 152.72 & 153.14 & -1.21 \\
\hline 147.42 & 147.84 & -1.43 & 152.82 & 153.24 & -1.25 \\
\hline 147.52 & 147.94 & -1.30 & 153.12 & 153.54 & -1.14 \\
\hline 147.62 & 148.04 & -1.41 & 153.24 & 153.66 & -1.26 \\
\hline 147.72 & 148.14 & -1.19 & 153.32 & 153.74 & -1.40 \\
\hline 147.82 & 148.24 & -1.03 & 153.42 & 153.84 & -1.41 \\
\hline 147.92 & 148.34 & -1.20 & 153.62 & 154.04 & -1.30 \\
\hline 148.02 & 148.44 & -1.32 & 153.72 & 154.14 & -1.43 \\
\hline 148.12 & 148.54 & -1.36 & 153.82 & 154.24 & -1.28 \\
\hline 148.23 & 148.65 & -1.26 & 153.92 & 154.34 & -1.22 \\
\hline 148.32 & 148.74 & -1.26 & 154.42 & 154.84 & -1.37 \\
\hline 148.42 & 148.84 & -1.25 & 154.52 & 154.94 & -1.42 \\
\hline
\end{tabular}

Note: Depths are sub-bottom depths and composite depths (MCD); see text.

glacials, isotope Stages 6 and 12, as documented by Berger et al. (this volume). Although this stage stands out remarkably in the Hole 806B record, and it also is a strong glacial in other high-resolution records, benthic isotope records indicate that it has lower amplitude and is similar in strength to isotope Stages 96 and 98 (Raymo et al., 1989; Shackleton et al., 1990). This difference between the planktonic and benthic records indicates that the planktonic oxygen isotope record from Hole $806 \mathrm{~B}$ to some extent is influenced by temperature overprints, despite the favorable location of Site 806.

The cooling trend seen in the Hole $806 \mathrm{~B}$ record started at about 3.7 Ma (Fig. 2), long before the emergence of intense Northern Hemisphere glaciation. Earlier studies have shown a cooling trend from about 3.1 Ma (based on the standard magnetic time scale), culminating with major equatorward shifts in the position of the oceanic polar fronts in both hemispheres and extensive glacial marine sedimentation close to the Gauss/Matuyama boundary (Shackleton et al., 1984; Backman and Pestiaux, 1986; Keigwin, 1987; Raymo et al., 1987; Hodell and Ciesielski, 1991). At 2.57 Ma the first major sedimentation of ice-rafted detritus (IRD) took place in the North Atlantic (Jansen et al., 1988; Jansen and Sjøholm, 1991), and glacials of similar magnitude as those in the late Quaternary appeared about $100,000 \mathrm{yr}$ later. Thus, the process of climatic deterioration in the late Pliocene is not a story of stepwise shifts of climate modes at about $2.5 \mathrm{Ma}$ (standard magnetic time scale), nor is it a clear-cut cooling trend. It is a history of both a cooling trend, with superimposed ice-volume and climate fluctuations of Milankovitch type, as well as a shift in the pattern of oxygen isotope fluctuations after about 2.85 (2.73) Ma (Stage 116). At some critical point, the cooling trend evolved into a system of repeated strong Northern Hemisphere glacial cycles, which further developed to a magnitude that allowed Northern Hemisphere glaciers to become large enough to export large quantities of icebergs to the North Atlantic followed by a series of large glacial stades.

\section{CYCLICITY OF OXYGEN ISOTOPE RECORD}

The visual impression of the $\delta^{18} \mathrm{O}$ signal is that it displays cyclical changes, and that the frequency pattern varies with time (Figs. 2 and 3 ). Thus, we can proceed on the assumption that we should see Milankovitch-type cyclicity in these data. To evaluate the frequency distribution of the record as well as the relation between isotope cyclicity and forcing functions, we performed evolutionary spectral analyses (see Mayer, 1991, and Mayer et al., this volume) of the record, using two different time scales. The effects of changing the time scales can be viewed in Figures 4 and 5 . The evolutionary spectra were produced by moving a 900,000 -yr window along the time series with steps of $90,000 \mathrm{yr}$. The large overlap ( $90 \%$ ) enables us to capture evolutionary trends in the frequency pattern of the record, although this approach has the disadvantage that potentially rapid shifts in the frequency distribution will be smoothed by the large overlap.

The pure biostratigraphic time scale used for the evolutionary spectra of Figure 4 shows very little energy connected with the 41-k.y. tilt frequency. For the period from 3 to $2 \mathrm{Ma}$, indications of spectral power related to the precession frequencies around $20 \mathrm{k} . \mathrm{y}$. are present. When we use the additional age information from the oxygen isotope stages and the age model of Shackleton et al. (1990), and with 5\% added to datums within the Gauss and Gilbert chrons (Fig. 5), we observe a clear concentration of variance centered close to 41 k.y. in the period between 3 and $2 \mathrm{Ma}$. The energy peak is, however, slightly away from the 41-k.y. target. This is the same effect that is documented for grain-size and GRAPE records by Mayer et al. (this volume). Neither of the time scales produce strong energy peaks in $\delta^{18} \mathrm{O}$ at $41 \mathrm{k} . \mathrm{y}$. in the period before $3 \mathrm{Ma}$. There is, however, some tendency toward more 41-k.y. power at the beginning of our time series around $4 \mathrm{Ma}$ (Figs. 5 and 6). Also present is an energy peak at about 35 k.y. in the interval after $3 \mathrm{Ma}$.

The emergence of stronger 41-k.y. cyclicity after 3 Ma can partly be a result of the better time control we obtain by adding the isotope stage control points to the age model in the 3-2 Ma period. Nevertheless, visual examination of the record in Figures 2 and 3 indicates, as mentioned above, that the mode of variation appears to shift from a high-frequency type to a mode with higher amplitudes and more consistent lower frequencies after approximately 2.85 (2.73) Ma, or isotope Stage 116, according to our age model. This is clearly reflected by the evolutionary spectra and indicates to us that at this point the climate system more easily responds to obliquity-related forcing, and that high-latitude ice-volume fluctuations, probably in the Northern Hemisphere, from this time on appear to be significant factors in the way the system responds to forcing. Shackleton et al. (1988) analyzed North Atlantic records from Site 552 and also documented low 41-k.y. power between 2.5 and $3.5 \mathrm{Ma}$.

The increased 41-k.y. sensitivity that emerged at about $2.85(2.73) \mathrm{Ma}$ must have been forced by some change in climate boundary conditions, 


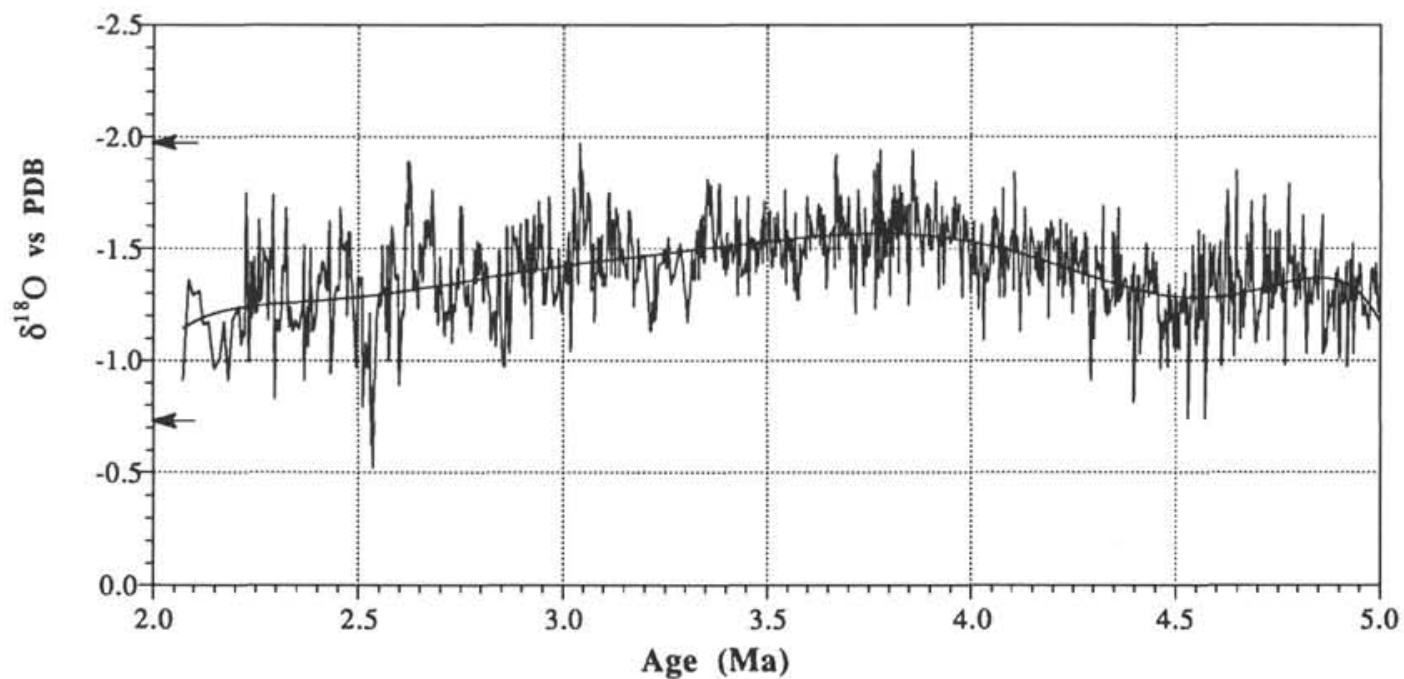

Figure 2. Oxygen isotope record from Hole 806B of G. sacculifer plotted vs. age, based on the Shackleton et al. (1990) age model. See text and accompanying paper by Mayer et al. (this volume). The line drawn through the data set is a polynomial fit to the data.

as no apparent change is present in the amplitude of the orbital forcing function (Berger and Loutre, 1991). The change in boundary conditions must particularly affect high-latitude regions as it produces a $41-k . y$. response related to obliquity, which has its strongest climatic effects at high latitudes (Short et al., 1990). Recent studies have focused on the effects of increased mountain building and uplift in Tibet/Himalaya and western North America on two important elements of the climate system: the global atmospheric circulation patterns and the atmospheric $\mathrm{CO}_{2}$ levels. Ruddiman and Raymo (1988) and Ruddiman and Kutzbach (1989) proposed that the atmospheric circulation changes provided by mountain uplift in these regions would enhance high northern latitude cooling. Raymo et al. (1988) also suggested that mountain building would enhance weathering and provide a $\mathrm{CO}_{2}$ draw-down mechanism by increasing oceanic alkalinity. Modeling experiments aimed at investigating the climatic effects of doubling atmospheric $\mathrm{CO}_{2}$ concentrations compared to preindustrial $\mathrm{CO}_{2}$ levels have in common that the strongest climate effects of changing atmospheric $\mathrm{CO}_{2}$ will be felt in the arcticsubarctic regions (see IPCC, 1990). Hence, both of the effects of mountain uplift form long-term forcing factors that tend to increase high-latitude climate sensitivity and are thus compatible with our results. The rather sharp shift in variability at about 2.85 (2.73) Ma may, therefore, be the result of a change in the sensitivity of the climate system to slow tectonic forcing. One way this may have operated is through the effects of the deep circulation, in which increased glaciation in the Northern Hemisphere may have suppressed the formation of northern-source deep water, which reduced oceanic heat in the Southern Ocean and impacted atmospheric $\mathrm{CO}_{2}$. Such responses were proposed by Hodell and Venz (in press) based on records from South Atlantic Site 704.

The closure of the Panama seaway at 4-3 Ma (Keigwin, 1982) did not have any clear impact on the frequency components of our record, but it may be a factor to consider when explaining the cooling trend after 3.7 Ma. A modeling experiment with the Hamburg geostrophic global ocean model indicates that one effect of the Panama closure was to enhance North Atlantic meridionality (Maier-Raimer et al., 1990), which may be an important factor controlling the moisture supply to feed waxing ice sheets.

Cross-spectral analyses that compare the Hole $806 \mathrm{~B} \delta^{18} \mathrm{O}$ record with $65^{\circ} \mathrm{N}$ summer insolation throughout the 5-2 Ma period show no coherency above the $80 \%$ significance level between the orbital forcing function (insolation) and the 41-k.y. frequency in the $\delta^{18} \mathrm{O}$ record (Fig. 6). The evolutionary spectra (Fig. 5) and the filtered results (Fig. 7) indicate a higher concentration of variance at 41 k.y. after $3 \mathrm{Ma}$ than before. The poor coherency may in part be a result of a poorly constrained age model, and we would expect to see some improvement in the power and coherency at the Milankovitch frequencies with an improved time scale. Also, a low signal-to-noise ratio caused by the lower amplitude of isotope variations before 3 Ma may contribute to this effect. The low coherency and low spectral power at the Milankovitch frequencies indicate, however, that the climate system in general was less susceptible to orbital forcing before $3 \mathrm{Ma}$ than afterward. The low coherency (Fig. 6) and low energy seen at the 41 - and 21 -k.y. filtered $\delta^{18} \mathrm{O}$ (Fig. 7) indicates that it will be difficult to improve the time scale by using the $\delta^{18} \mathrm{O}$ record as a tuning tool. The grain-size and GRAPE records appear, on the contrary, to have stronger concentrations of variance at orbital frequencies during the period studied (Mayer et al., this volume). These records also display clear 41-k.y. cyclicity before $3 \mathrm{Ma}$ and are more suitable for tuning purposes. Thus, some elements of the climate system respond to obliquity forcing while others, such as ice volume, apparently do not. According to Mayer et al. (this volume), the grain-size and density variations on the Ontong Java Plateau are most probably caused by variations in deep-water flow. Thus, although deep-water variability responds to high-latitude forcing at the obliquity frequency, a much more subdued and less obvious response in ice volume to obliquity-related forcing before $2.85 \mathrm{Ma}$ is present. Deep-water variability may have originated in the highlatitude North Atlantic region, as good evidence exists for continuous strong formation of northern source water before the glacial onset at about $2.7 \mathrm{Ma}$ (M. Raymo, pers. comm., 1992). The lack of significant ice volumes in the Northern Hemisphere before $2.85 \mathrm{Ma}$ may have prevented a similar response in ice volume $\left(\delta^{18} \mathrm{O}\right)$.

The $\delta^{18} \mathrm{O}$ record, in contrast, appears to be dominated by high-frequency variability in the 5-3 Ma period, although some 41-k.y. power appears at 5 to $4.2 \mathrm{Ma}$ (Fig. 5), which was a time of larger $\delta^{18} \mathrm{O}$ amplitude and probably higher ice volumes (see above). Better age control may focus the high-frequency variance better to the precessional frequencies, but it is obvious that much of the variance is located at frequencies higher than the Milankovitch frequencies, either reflecting noise in the record or some statistically significant variance at higher frequencies. These may be harmonics or combination frequencies of the precessional frequencies. One alternative is that we observe half-frequencies reflecting the dual passage of precessional forcing across the equator (i.e., passing the equator twice in one cycle from one extreme end to the opposite and back again). Because precession is the dominant insolation forcing in the tropics, the tendency for high-frequency variance during the 5-3 Ma period 

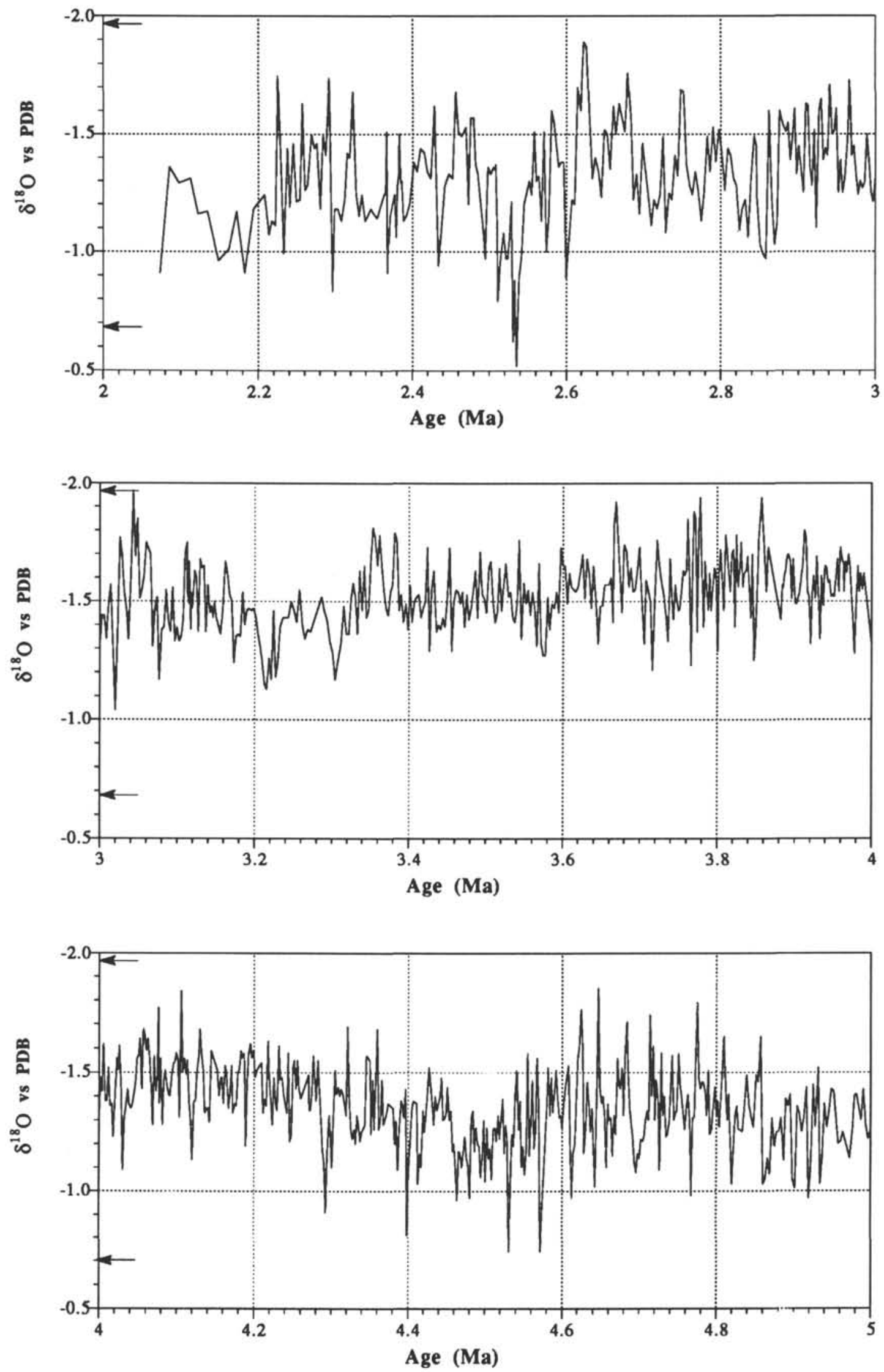

Figure 3. Oxygen isotope record from Hole 806B of G. sacculifer on an expanded scale (from 2 to $5 \mathrm{Ma}$ ). Arrows denote late Quaternary range (Berger et al., this volume). 


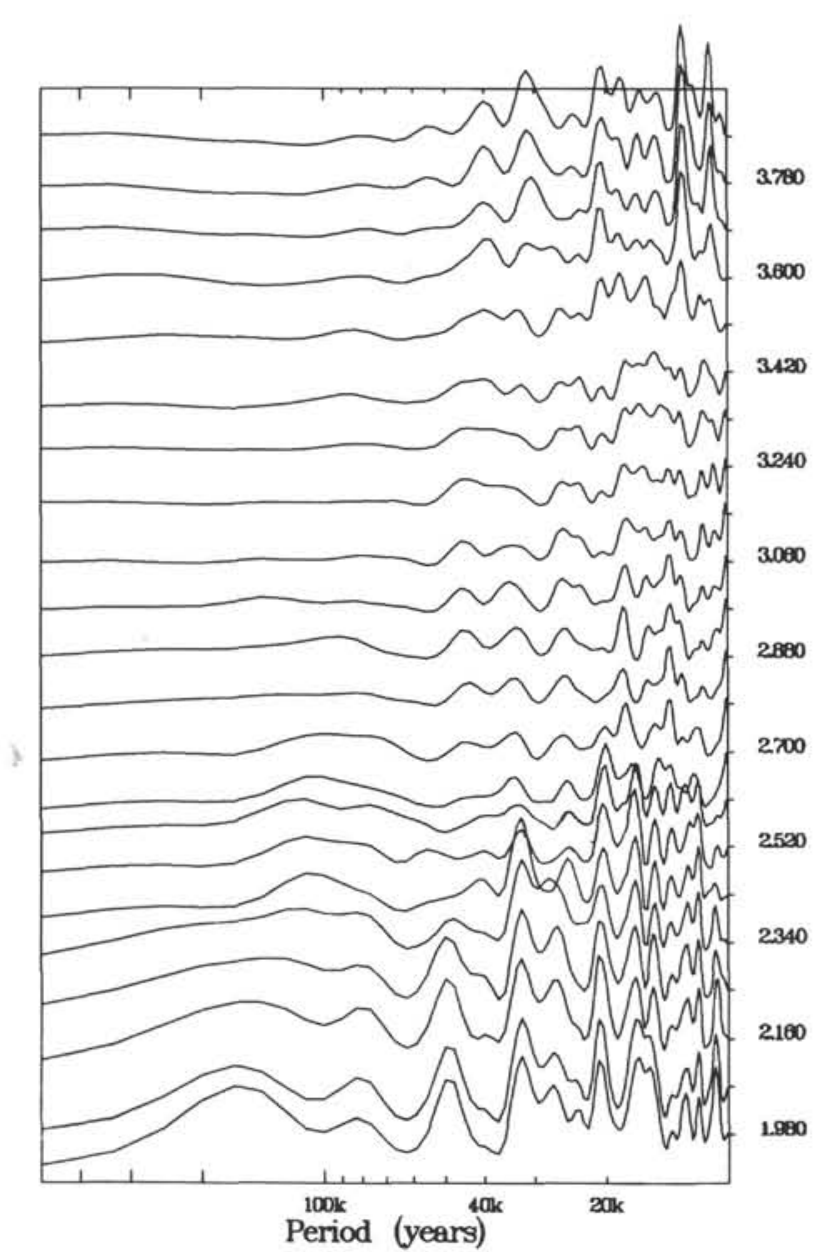

Figure 4. Evolutionary spectra for the Hole $806 \mathrm{~B} \delta^{18} \mathrm{O}$ record, using the pure biostratigraphic time scale. Each spectrum represents a spectral analysis of a 900,000 -yr segment of the record. For each new spectrum, this $900,000-\mathrm{yr}$ window was moved by $90,000 \mathrm{yr}$, thus producing a $90 \%$ overlap with the preceding spectrum. The spectra are energy conserving. The age given on the right-hand side indicates the age of the beginning of each 900,000-yr segment.

makes it possible that the dominant factor driving the $\delta^{18} \mathrm{O}$ variance in that period was local temperature effects driven by changes in equatorial wind stress. Thus, we may see two modes of variability: (1) when ice volumes were large on the average, with ice-volume variability forced by high-latitude insolation changes in the 41-k.y. band; and (2), a mode operating at times of lower ice volumes, with high-frequency variations of sea-surface temperature forced by the effects of precession on the equatorial system. This pattern is different from that shown by Joyce et al. (1990) in a study of planktonic foraminiferal $\delta^{18} \mathrm{O}$ from ODP Site 625 in the Gulf of Mexico. They found 41 k.y. to be the dominant frequency throughout the last 5.35 m.y., with high concentrations of 41-k.y. power at 4.5-5 Ma and around $3.5 \mathrm{Ma}$. The increase in $41-\mathrm{k} . \mathrm{y}$. power around $2.5 \mathrm{Ma}$ is also documented in their record. However, the higher concentration of 41-k.y. variance in their record may, to a large extent, be ascribed to the fact that their record was tuned to obliquity.

Figure 8 indicates that variance at the $21-k . y$. frequency also increased after $3 \mathrm{Ma}$. The cross spectral analysis shows that this is not coherent with insolation. Figure 9 illustrates clearly that the modulation of the 21-k.y. filtered output of $\delta^{18} \mathrm{O}$ is very different from the modulation of insolation documented by the record we obtain by filtering insolation with the same filter. Therefore, the $\delta^{18} \mathrm{O} 21-\mathrm{k} . \mathrm{y}$. cyclicity is, at least with the time scale we now have at hand, not

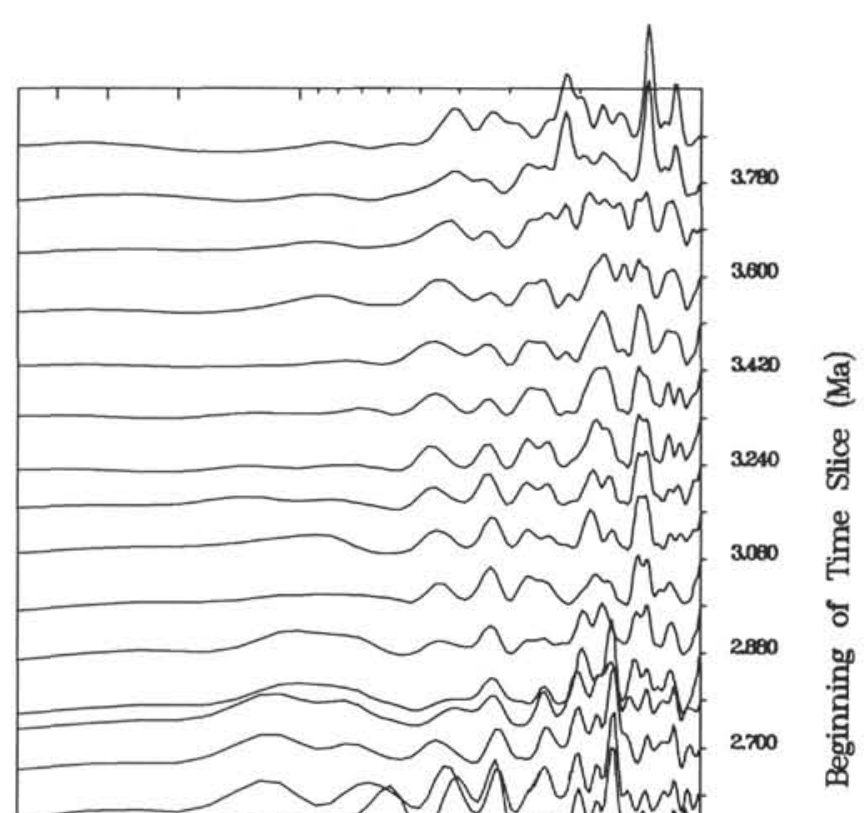
et al. (1990). See text and accompanying paper by Mayer et al. (this volume).

linearly related to precessional forcing. Although improvements in the time scale may lead to improved coherency with orbital forcing, it may continue to be low, as judged by the comparison in Figure 8, which shows very little comparable modulation between the filtered insolation and the filtered $\delta^{18} \mathrm{O}$. Hagelberg and Pisias (1990) documented in a study, covering the same period as our study, that SST and planktonic $\delta^{18} \mathrm{O}$ variations in the tropical eastern Pacific were nonlinearly related to orbital forcing. Although insolation and SST showed low coherence, Hagelberg and Pisias (1990) were able to show that the amplitude modulations of precession and the climate signals at $23 \mathrm{k} . \mathrm{y}$. and other frequencies were highly coherent. This nonlinear behavior of SST and $\delta^{18} \mathrm{O}$ is different from the linearity documented in Pleistocene records (Imbrie et al., 1984); it indicates clear differences in the way the climate system responds to orbital forcing between the Pliocene and the Pleistocene. This change may also reflect a shift in the processes that govern the variations in equatorial planktonic $\delta^{18} \mathrm{O}$, from a system largely governed by lowlatitude processes driving SST variations when Northern Hemisphere ice volumes were low, to becoming more influenced by high- and mid-latitude variations in ice extent.

\section{CONCLUSIONS}

The detailed Pliocene oxygen isotope record from the Ontong Java Plateau documents major long- and short-term climate changes. The results show periods of enhanced ice volume at 4.6 to $4.3 \mathrm{Ma}$ and after 2.85 (2.73) Ma, a long-term warming trend 4.3-3.9 Ma, and a distinct 
cooling trend that was initiated at $3.7 \mathrm{Ma}$ and progressed through the initiation of large-scale Northern Hemisphere glaciation after 2.85 (2.73) Ma. The periods of high average ice volumes also show the highest $\delta^{18} \mathrm{O}$ amplitudes.

The pattern of climate cyclicity changed markedly at about 2.85 (2.73) Ma. The earlier times were mainly reflected by high-frequency variability at the precessional frequencies or even higher frequencies, pointing to low-latitude processes as a main controlling factor driving planktonic $\delta^{18} \mathrm{O}$ variability in this period. Some $41-\mathrm{k} . \mathrm{y}$. power is apparent in the record from 5 to $4 \mathrm{Ma}$, indicating a time of some high-latitude climate sensitivity. The high-frequency variability is not coherent with insolation and points to strong nonlinearity in the way the climate system responded to orbital forcing before the onset of large-scale Northern Hemisphere glaciation.

After $3 \mathrm{Ma}$, stronger 41-k.y. cyclicity appears in the record, coherent with orbital forcing at this frequency. The shift in pattern is most clear around 2.85 (2.73) Ma, some time before the most dramatic spread of Northern Hemisphere ice sheets. This indicates that high-latitude processes from this point on tend to take over and most strongly influence the $\delta^{18} \mathrm{O}$ record, which now reflects ice-volume fluctuations related to the climatic effects of obliquity forcing on the seasonality of high-latitude areas, most probably in the Northern Hemisphere.

The cyclicity of the record appears to be unaffected by the closure of the Panama gateway, whereas the cooling trend after 3.7 may be related to this tectonic event.

The general Pliocene trend is that high-latitude climate sensitivity and instability was increasing, and the causal factors producing the intensified glacial cyclicity during the Pliocene must be factors that enhance cooling and climate sensitivity in the subarctic areas.

\section{ACKNOWLEDGMENTS}

We thank Odd Hansen and Rune Søraas for their dedicated operation of the mass spectrometer and for sample preparation, Karl Johan Karlsen and Gerd Solbakken for overseeing preparation of the large number of samples, and our Leg 130 shipboard colleagues for their cheerful tenure at the sampling table. We are grateful to Steven Bloomer, who helped us with the statistical analyses, and to André Berger for giving us the insolation calculations. Warm thanks goes to Captain Oonk and the crew of the JOIDES Resolution and the scientific support staff on the ship for providing a highly professional and friendly cruise. Reviews by Dave Hodell and Maureen Raymo helped us improve the original manuscript, and information from Maureen Raymo and Nick Shackleton was very helpful in sorting out time-scale problems.

\section{REFERENCES}

Backman, J., and Pestiaux, P., 1986. Pliocene discoaster abundance variations, Deep Sea Drilling Project, Site 606: biochronology and paleoenvironmental implications. In Kidd, R., Ruddiman, W.F., et al., Init. Repts. DSDP, 94, Pt. 2: Washington (U.S. Govt. Printing Office), 903-909.

Berger, A., and Loutre, M.F., 1991. Insolation values for the climate of the last 10 million years. Quat. Sci. Rev., 10:297-317.

Berger, W.H., Killingley, J.S., and Vincent, E., 1978. Stable isotopes in deep sea carbonates: box core ERDC92, west equatorial Pacific. Oceanol. Acta, 1:203-216.

Chapell, J., and Shackleton, N.J., 1986. Oxygen isotopes and sea level. Nature, 324:137-140.

Fairbanks, R.G., 1989. A 17,000-year glacio-eustatic sea level record: influence of glacial melting rates on the Younger Dryas event and deep ocean circulation. Nature, 342:637-642.

Fairbanks, R.G., Sverdlove, M., Free, R., Wiebe, P.H., and Bé, A.W.H., 1982. Vertical distribution and isotopic composition of living planktonic foraminifera from the Panama Basin. Nature, 298:841-844.

Hagelberg, T.K., and Pisias, N., 1990. Nonlinear response of Pliocene climate to orbital forcing: evidence from the eastern equatorial Pacific. Paleoceanography, 5:595-618.
Hodell, D.A., and Ciesielski, P.F., 1991. Stable isotope and carbonate stratigraphy of the late Pliocene and Pleistocene of Hole 704A: eastern subantarctic South Atlantic. In Ciesielski, P.F., Kristoffersen, Y., et al., Proc. ODP, Sci. Results, 114: College Station, TX (Ocean Drilling Program), 409-435.

Hodell, D.A., and Venz, K., in press. Toward a high-resolution stable isotopic record of the Southern Ocean during the Plio-Pleistocene (4.8 to $0.8 \mathrm{Ma}$ ). Antarc. Res. Ser.

Imbrie, J., Hays, J.D., Martinson, D.G., McIntyre, A., Mix, A.C., Morley, J.J., Pisias, N.G., Prell, W.L., and Shackleton, N.J., 1984. The orbital theory of Pleistocene climate: support from a revised chronology of the marine delta $\delta^{18} \mathrm{O}$ record. In Berger, A., Hays, J., Imbrie, J., Kukla, G., and Saltzman, B. (Eds.), Milankovitch and Climate (Pt. 1): Dordrecht (D. Reidel), 269-305.

IPCC (Intergovernmental Panel on Climatic Change), 1990. Houghton, J.T., Jenkins, G.J., and Ephraums, J.J. (Eds.), The IPCC Scientific Assessment: Cambridge, UK (Press Syndicate of the Univ. of Cambridge).

Jansen, E., Bleil, U., Henrich, R., Kringstad, L., and Slettemark, B., 1988. Paleoenvironmental changes in the Norwegian Sea and the Northeast Atlantic during the last $2.8 \mathrm{Ma}$ : DSDP/ODP Sites $610,642,643$ and 644 . Paleoceanography, 3:563-581.

Jansen, E., and Sjøholm, J., 1991. Reconstruction of glaciation over the past 6 million years from ice-borne deposits in the Norwegian Sea. Nature, 349:600-604.

Jansen, E., Sjøholm, J., Bleil, U., and Erichsen, J.A., 1990. Neogene and Pleistocene glaciations in the Northern Hemisphere and late MiocenePliocene global ice volume fluctuations: evidence from the Norwegian Sea. In Bleil, U., and Thiede, J. (Eds.), Geologic History of the Polar Oceans-Arctic versus Antarctic: Dordrecht (Kluwer Publ.).

Joyce, J.E., Tjalsma, L.R., and Prutzman, J.M., 1990. High resolution planktic stable isotope record and spectral analysis for the last 5.35 m.y.: Ocean Drilling Program Site 625 Northeast Gulf of Mexico. Paleoceanography, 5:507-530.

Keigwin, L.D., Jr., 1982. Isotopic paleoceanography of the Caribbean and East Pacific: role of Panama uplift in late Neogene time. Science, 217:350-353.

, 1987. Pliocene stable isotope record of Deep Sea Drilling Project Site 606: sequential events of ${ }^{18} \mathrm{O}$ enrichment beginning at $3.1 \mathrm{Ma}$. In Ruddiman, W.F., Kidd, R.B., Thomas, E., et al., Init. Repts. DSDP, 94, Pt. 2: Washington (U.S. Govt. Printing Office), 911-920.

Labeyrie, L.D., Duplessy, J.C., and Blanc, P.L., 1987. Variations in mode of formation and temperature of oceanic deep waters over the past 125,000 years. Nature, 327:477-482.

Maier-Raimer, E., Mikolajevicz, U., and Crowley, T.J., 1990. Ocean GCM experiment with an open central American isthmus. Paleoceanography, 5:49-366.

Mayer, L.A., 1991. Extraction of high-resolution carbonate data for paleoclimate reconstruction. Nature, 352:148-150.

Ravelo, A.C., 1991. Reconstructing the tropical Atlantic seasonal thermocline using planktonic foraminifera [Ph.D. thesis]. Columbia Univ., New York, NY.

Raymo, M.E., Ruddiman, W.F., Backman, J., Clement, B.M., and Martinson, D.J., 1989. Late Pliocene variation in Northern Hemisphere ice-sheets and North Atlantic deep water circulation. Paleoceanography, 4:413-446.

Raymo, M.E., Ruddiman, W.F., and Clement, B.M., 1987. Pliocene-Pleistocene paleoceanography of the North Atlantic at Deep Sea Drilling Project Site 609. In Ruddiman, W.F., Kidd, R.B., Thomas, E., et al., Init. Repts. DSDP, 94, Pt. 2: Washington (U.S. Govt. Printing Office), 895-901.

Raymo, M.E., Ruddiman, W.F., and Froelich, P.N., 1988. The influence of late Cenozoic mountain building on oceanic geochemical cycles. Geology, 16:649-653.

Ruddiman, W.F., and Kutzbach, J.E., 1989. Forcing of late Cenozoic Northern Hemisphere climate by plateau uplift in southern Asia and the American west. J. Geophys. Res., 94:379-391.

Ruddiman, W.F., and Raymo, M.E., 1988. Northern Hemisphere climate regimes during the last $3 \mathrm{Ma}$ : possible tectonic connections. Philos. Trans. R. Soc. London, Ser. B, 318:411-430.

Ruddiman, W.F., Raymo, M.E., and McIntyre, A., 1986. Matuyama 41,000year cycles: North Atlantic Ocean and Northern Hemisphere ice sheets. Earth Planet. Sci. Lett., 80:117-129.

Ruddiman, W.F., Raymo, M.E., Martinson, D.G., Clement, B.M., and Backman, J., 1989. Pleistocene evolution: Northern Hemisphere ice sheets and North Atlantic Ocean. Paleoceanography, 4:353-412.

Shackleton, N.J., Backman, J., Zimmermann, H., Kent, D.V., Hall, M.A., Roberts, D.G., Schnitker, D., Baldauf, J., Desprairies, A., Homrighausen, R., Huddlestun, P., Keene, J.B., Kaltenbach, A.J., Krumsick, K.A.O., 
Morton, A.C., Murray, J.W., and Westberg-Smith, J., 1984. Oxygen isotope calibration of the onset of ice-rafting and history of glaciation in the North Atlantic region. Nature, 307:620-623.

Shackleton, N.J., Berger, A., and Peltier, W.R., 1990. An alternative astronomical calibration of the lower Pleistocene timescale based on ODP Site 677. Trans. R. Soc. Edinburgh, Earth Sci., 81:251-261.

Shackleton, N.J., Imbrie, J., and Pisias, N.G., 1988. The evolution of oceanic oxygen isotope variability in the North Atlantic over the past three million years. Philos. Trans. R. Soc. London, Ser. B, 318:679-688.

Shackleton, N.J., and Opdyke, N.D., 1973. Oxygen isotope and paleomagnetic stratigraphy of equatorial Pacific core V28-238: oxygen isotope temperatures and ice volumes on a $10^{5}$ and $10^{6}$ year scale. Quat. Res., 3:39-55.

A
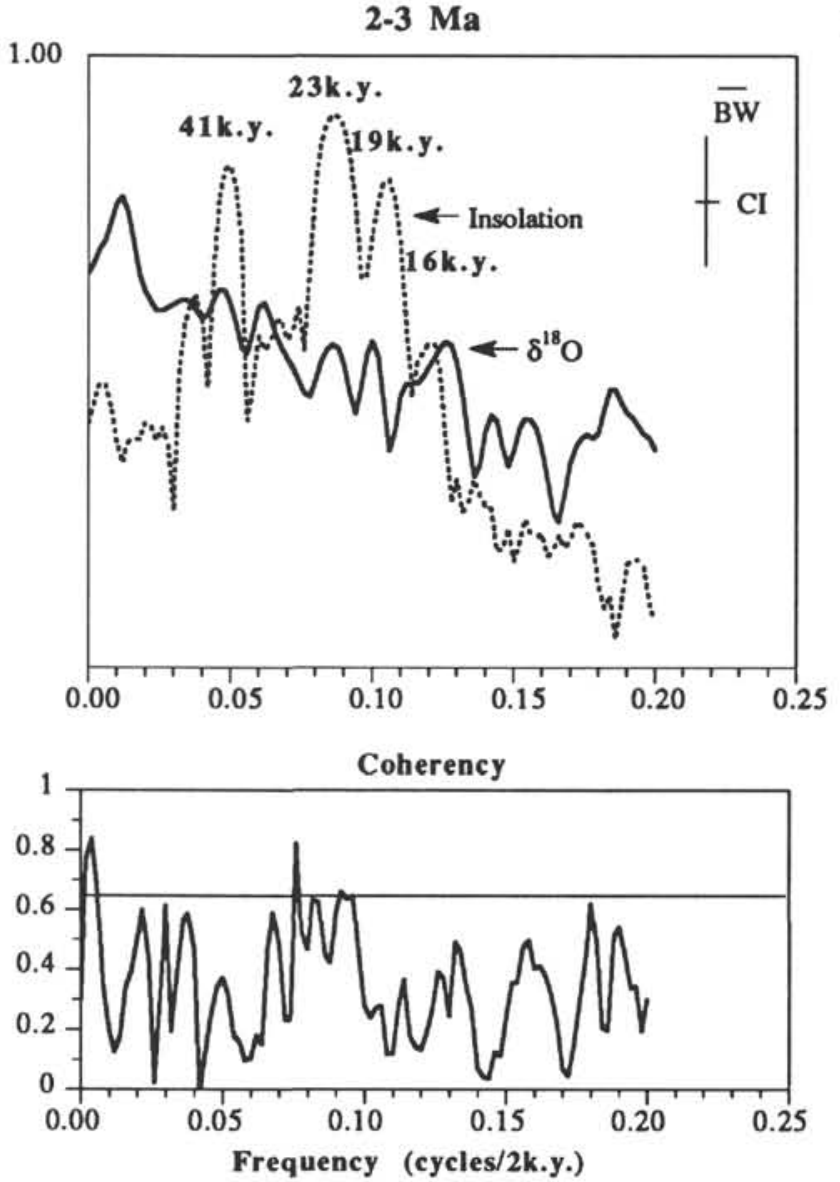

Short, D.A., Mengel, J.G., Crowley, T.J., Hyde, W.T., and North, G.R., 1990. Filtering of Milankovitch cycles by Earth's geography. Quat. Res. 35:157-173.

Webb, P.N., and Harwood, D.M., 1991. Late Cenozoic glacial history of the Ross Embayment, Antarctica. Quat. Sci. Rev., 10:215-224.

Date of initial receipt: 25 November 1991

Date of acceptance: 22 June 1992

Ms 130B-028

B

1.00
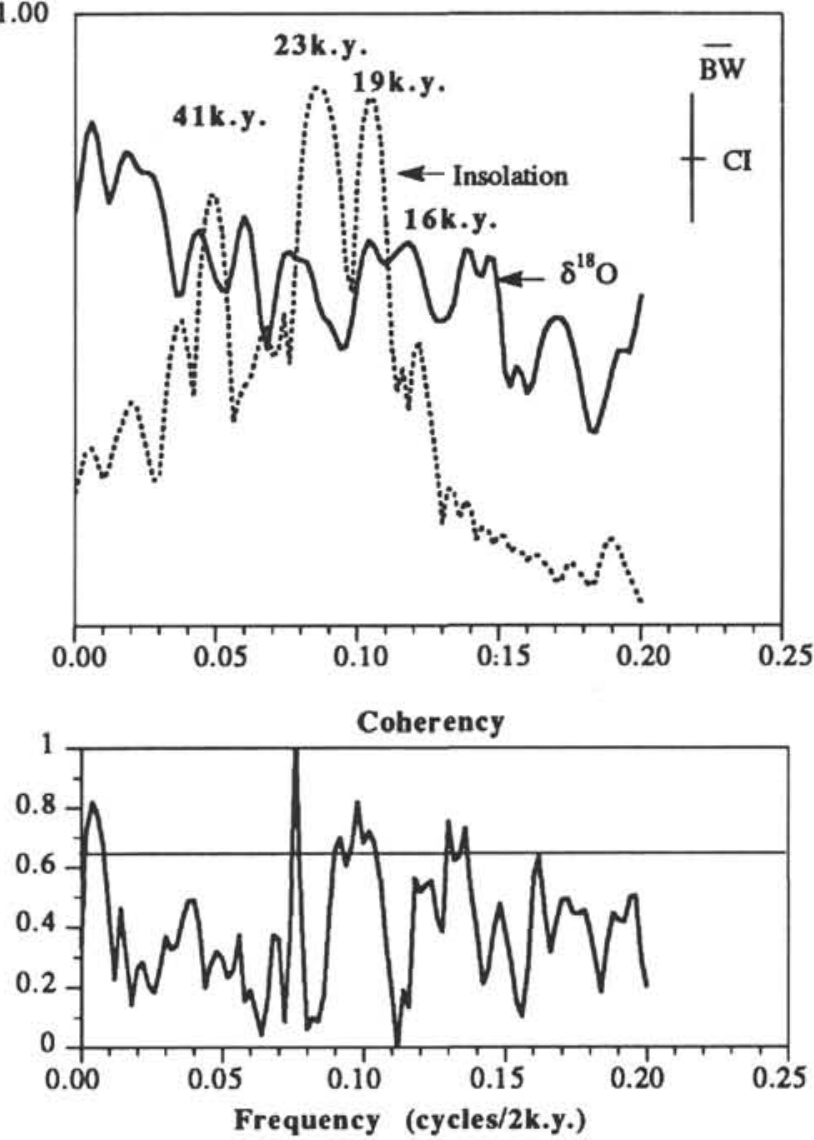

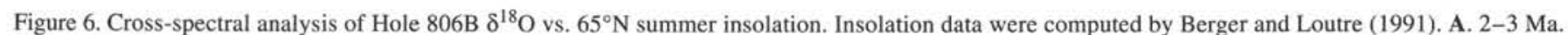

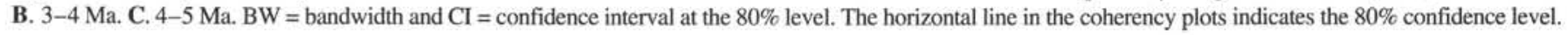


C
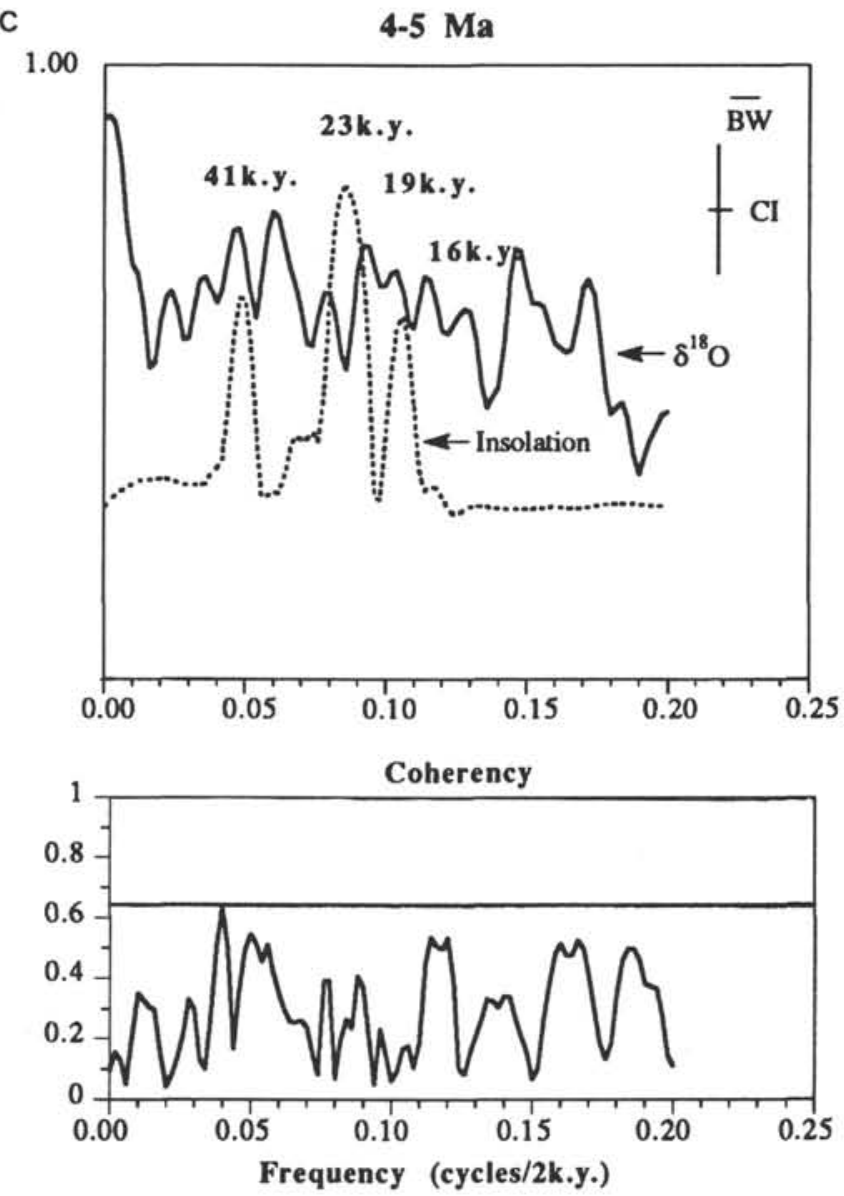

Figure 6 (continued).

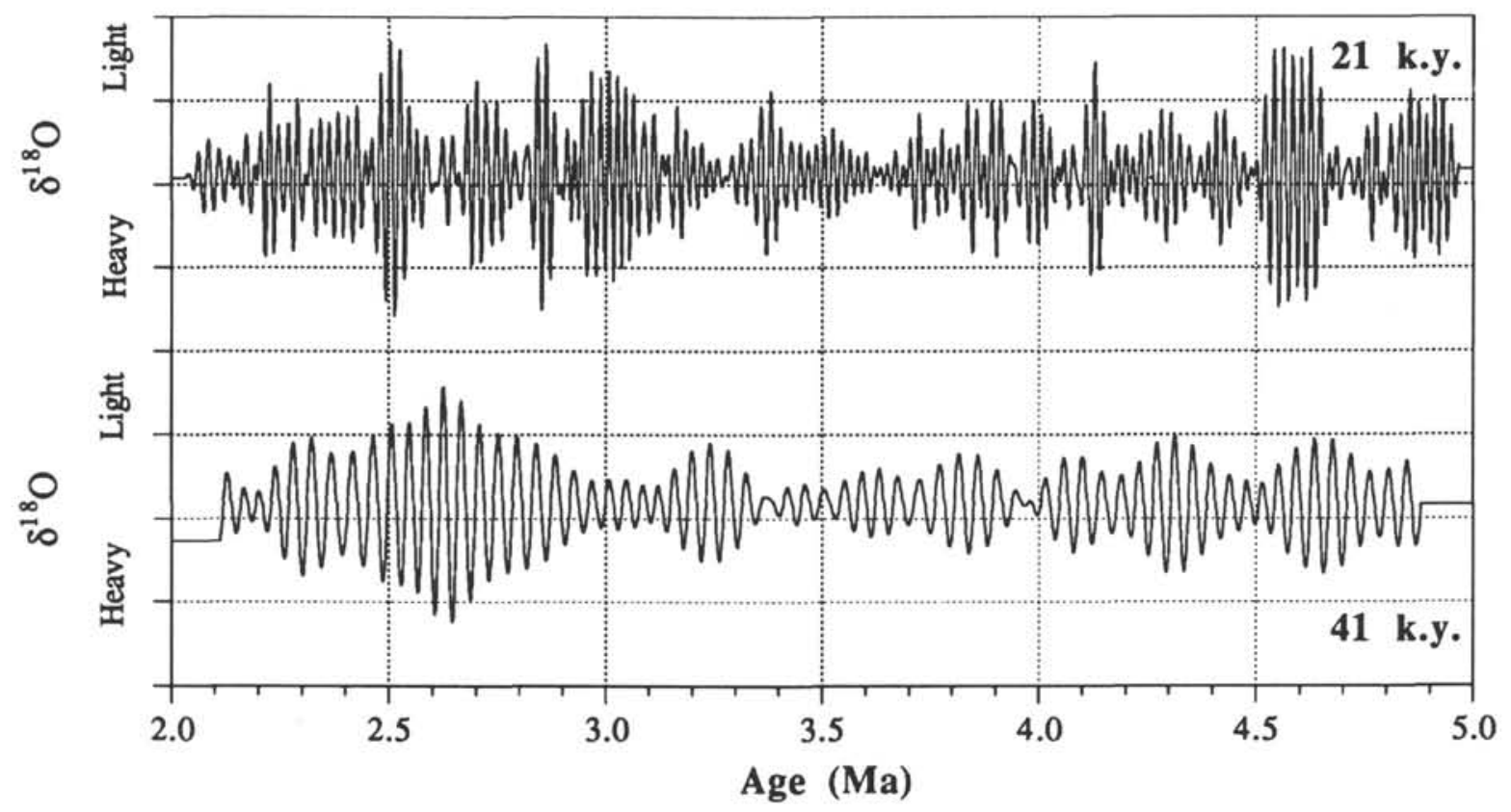

Figure 7. Hole $806 \mathrm{~B} \delta^{18} \mathrm{O}$ record filtered by band-pass filters with central frequencies at 41 and 21 k.y. 


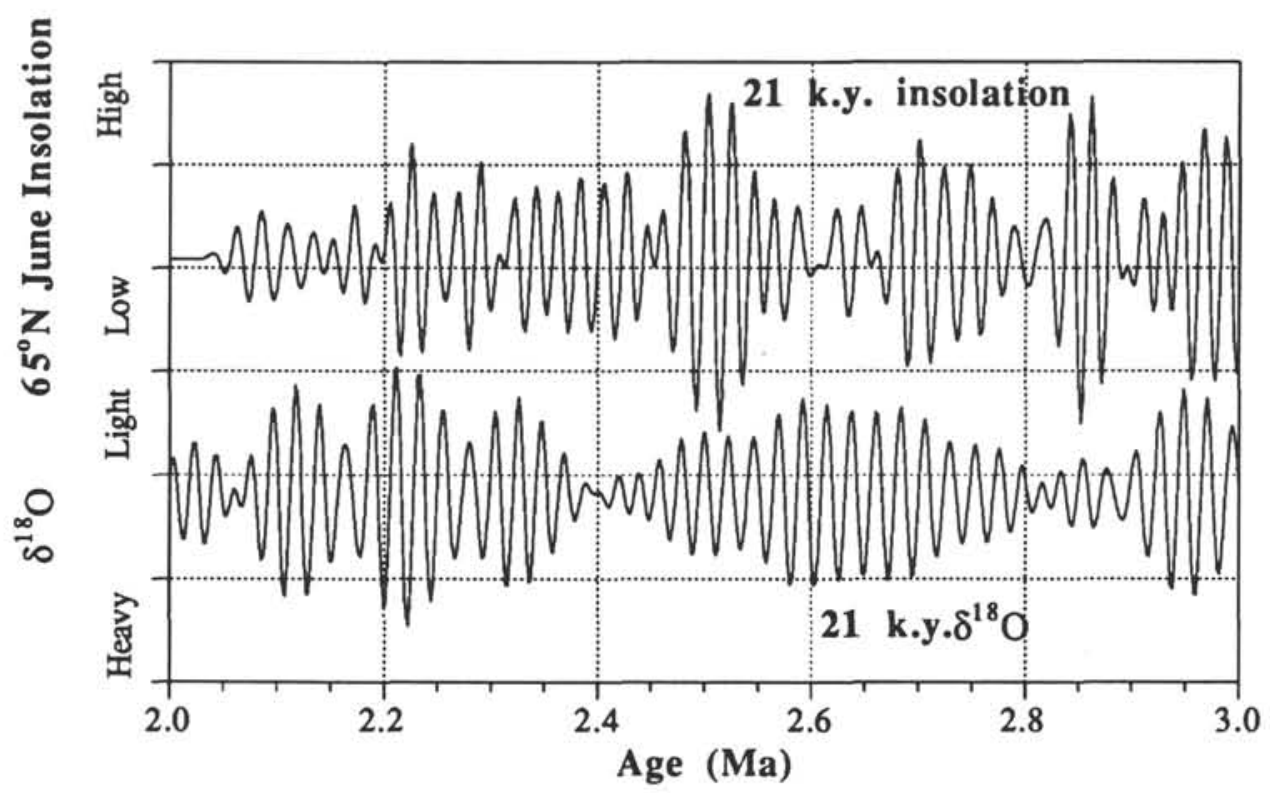

Figure 8 . Hole $806 \mathrm{~B} \delta^{18} \mathrm{O}$ and $65^{\circ} \mathrm{N}$ summer insolation filtered by a filter with a central frequency of 21 k.y. The same filter was used in Figure 7. The record shows output for the 2-3 Ma interval. 